\title{
Type Ic supernovae from the (intermediate) Palomar Transient Factory ${ }^{\star}$
}

\author{
C. Barbarino ${ }^{1}$, J. Sollerman ${ }^{1}$, F. Taddia ${ }^{1}$, C. Fremling ${ }^{2}$, E. Karamehmetoglu ${ }^{3}$, I. Arcavi ${ }^{4,5}$, A. Gal-Yam ${ }^{6}$, R. Laher ${ }^{7}$, \\ S. Schulze ${ }^{6}$, P. Wozniak ${ }^{8}$, and Lin Yan $^{9}$
}

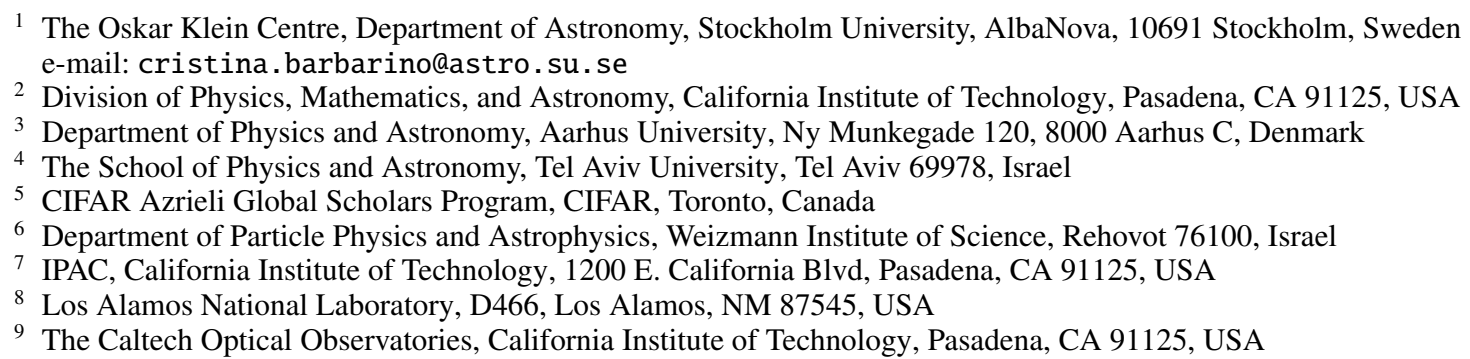

Received 10 July 2020 / Accepted 10 November 2020

\begin{abstract}
Context. Type Ic supernovae represent the explosions of the most stripped massive stars, but their progenitors and explosion mechanisms remain unclear. Larger samples of observed supernovae can help characterize the population of these transients.

Aims. We present an analysis of 44 spectroscopically normal Type Ic supernovae, with focus on the light curves. The photometric data were obtained over 7 years with the Palomar Transient Factory and its continuation, the intermediate Palomar Transient Factory. This is the first homogeneous and large sample of SNe Ic from an untargeted survey, and we aim to estimate explosion parameters for the sample.

Methods. We present K-corrected Bgriz light curves of these SNe, obtained through photometry on template-subtracted images. We performed an analysis on the shape of the $r$-band light curves and confirmed the correlation between the rise parameter $\Delta m_{-10}$ and the decline parameter $\Delta m_{15}$. Peak $r$-band absolute magnitudes have an average of $-17.71 \pm 0.85$ mag. To derive the explosion epochs, we fit the $r$-band lightcurves to a template derived from a well-sampled light curve. We computed the bolometric light curves using $r$ and $g$ band data, $g-r$ colors and bolometric corrections. Bolometric light curves and Fe II $\lambda 5169$ velocities at peak were used to fit to the Arnett semianalytic model in order to estimate the ejecta mass $M_{\mathrm{ej}}$, the explosion energy $E_{\mathrm{K}}$ and the mass of radioactive nickel $M\left({ }^{56} \mathrm{Ni}\right)$ for each $\mathrm{SN}$.

Results. Including $41 \mathrm{SNe}$, we find average values of $\left\langle M_{\mathrm{ej}}\right\rangle=4.50 \pm 0.79 M_{\odot},\left\langle E_{K}\right\rangle=1.79 \pm 0.29 \times 10^{51} \mathrm{erg}$, and $\left\langle M_{56 \mathrm{Ni}}\right\rangle=$ $0.19 \pm 0.03 M_{\odot}$. The explosion-parameter distributions are comparable to those available in the literature, but our large sample also includes some transients with narrow and very broad light curves leading to more extreme ejecta masses values.
\end{abstract}

Key words. supernovae: general

\section{Introduction}

Core-collapse supernovae (CC SNe) are the explosions of massive stars $\left(\gtrsim 8 M_{\odot}\right)$ which undergo gravitational collapse of the core at the end of their lifes. Their classification relies on the presence/absence of some spectroscopic features (e.g., Filippenko 1997; Gal-Yam 2017).

When they lack, partially or totally, hydrogen $(\mathrm{H})$ or helium $(\mathrm{He})$ they are called stripped envelope $\mathrm{SNe}$ (SE SNe). In this class we find $\mathrm{SNe} \mathrm{IIb}, \mathrm{Ib}$ and Ic. Type Ib SNe show no H but $\mathrm{He}$ in their spectra. SNe IIb show an initial signature of $\mathrm{H}$ at peak, which then disappears over time as their spectra become similar to those of Type Ib SNe. Supernovae Type Ic are the ones which lack both $\mathrm{H}$ and He. Two main scenarios have been proposed as progenitor systems of SE SNe: (i) single and

\footnotetext{
* Light curves are only available at the CDS via anonymous ftp to cdsarc.u-strasbg. fr (130.79.128.5) or via http://cdsarc. u-strasbg.fr/viz-bin/cat/J/A+A/651/A81
}

massive Wolf-Rayet (WR) stars that lose their outer envelopes through radiation-driven stellar winds (Begelman \& Sarazin 1986; Woosley et al. 1995), and (ii) lower mass stars in binary systems characterized by mass transfer (Wheeler \& Levreault 1985; Podsiadlowski et al. 1992; Yoon et al. 2010). It is still a matter of debate whether one or both of these progenitor channels can explain the observed SE SN population.

The estimated masses of the ejecta for SE SNe seem to favor the lower mass binary star scenario, rather than very massive single WR stars (Eldridge et al. 2013; Lyman et al. 2016; Cano 2013); and for example the data for the individual Type Ib SN iPTF13bvn seem to be more consistent with a binary system (Cao et al. 2013; Bersten et al. 2014; Fremling et al. 2014, 2016; Eldridge et al. 2015).

However, some SE SNe do seem to be originating from very massive stars (e.g., SN $2011 \mathrm{bm}$, Valenti et al. 2012; OGLE-2014-SN-131, Karamehmetoglu et al. 2017; LSQ14efd, Barbarino et al. 2017) and thus favor a single progenitor system. 


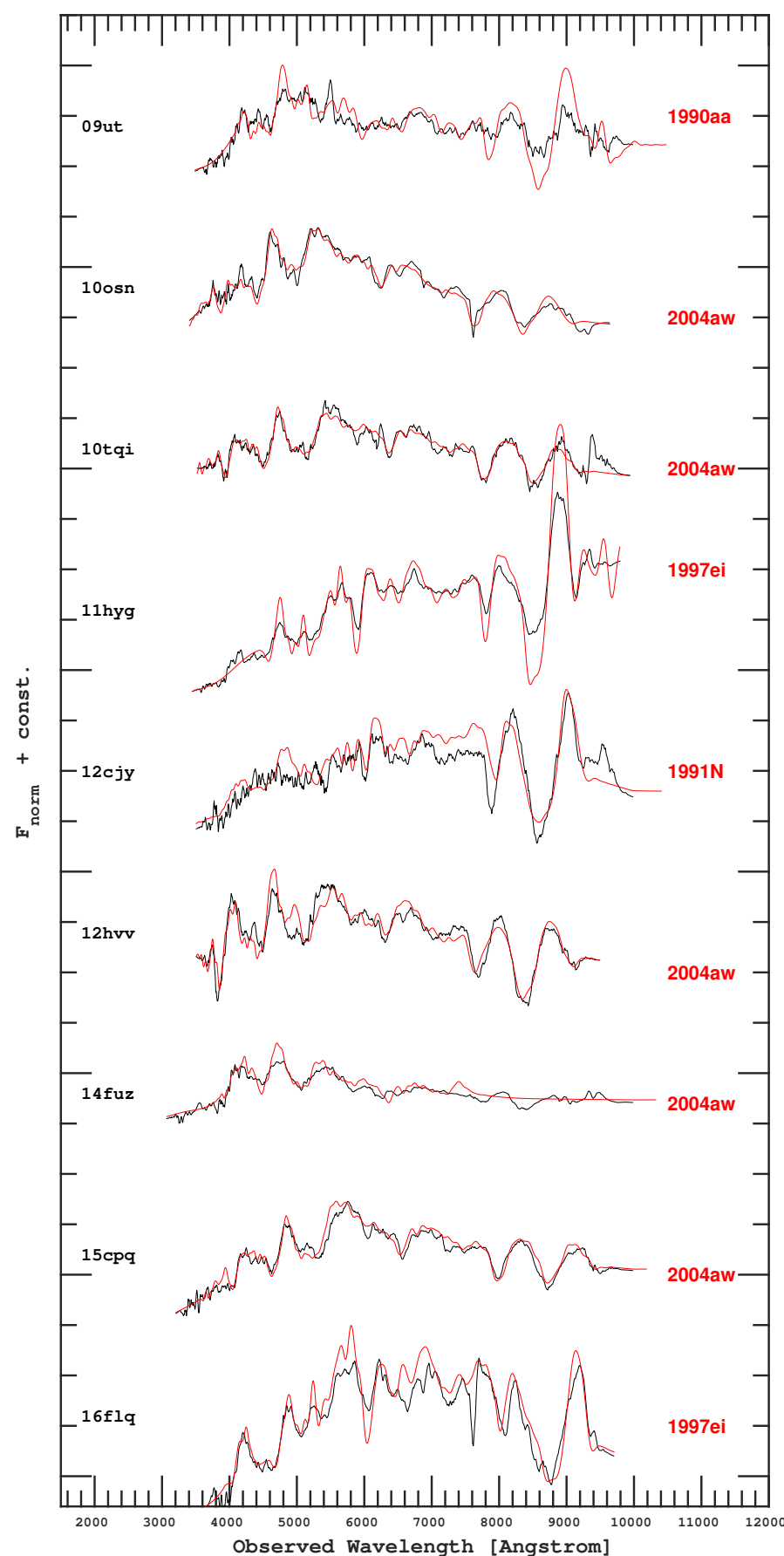

Fig. 1. Examples of $9 \mathrm{SNe}$ from the sample and their classification through the SNID package.

To complicate the picture we can have broad-lined Type Ic SNe that shows spectra of SNe Ic (e.g., SN 2003jd, Valenti et al. 2008) as well as super-luminous SNe that spectroscopically resemble SNe Ic (e.g., SN 2010gx, Pastorello et al. 2010).

The photometric samples available in the literature either refer to SE SNe (Lyman et al. 2016; Prentice et al. 2016, 2019; Taddia et al. 2018b) or to SNe Ibc (Drout et al. 2011; Taddia et al. 2015), including both SNe Ib and Ic. A major sample of merely SNe Ic (or Ib) is not available. Thanks to the untargeted surveys, the Palomar Transient Factory (PTF, Rau et al. 2009; Law et al. 2009) and its continuation, the intermediate Palomar Transient Factory (iPTF, Kulkarni 2013), we can here present optical observations of a large (44 objects) and

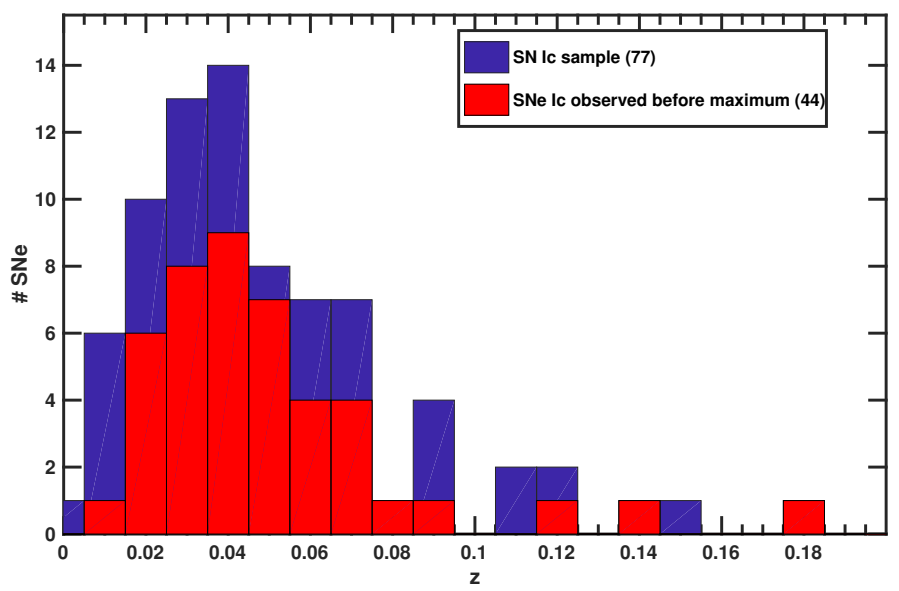

Fig. 2. Redshift distribution of all (i)PTF SNe Ic (blue) and of the 44 $\mathrm{SNe}$ Ic discovered before peak (red). The latter constitute the sample analyzed in this work.

homogeneous sample of spectroscopically normal SNe Ic. This enables a study of the properties of the SN population and their progenitor stars.

The paper is organized as follow: in Sect. 2 we present the sample while the photometry and the data reduction are presented in Sect. 3. The analysis of the light curves is discussed in Sect. 4. Bolometric light curves are presented in Sect. 5. The spectra and the analysis of the velocities are shown in Sect. 6. The explosion parameters are estimated in Sect. 7. The results are discussed, summarized and compared with the literature in Sect. 8.

\section{The sample}

During the 7 years of activity of PTF and iPTF, a total number of $60 \mathrm{SNe}$ Ic and $17 \mathrm{SNe}$ Ibc were discovered and followed up. Later in the this section we explain why and how this is narrowed down to $44 \mathrm{SNe}$. The classification of these objects was based on spectroscopy and performed using the Supernova Identification code (SNID, Blondin \& Tonry 2007) with the addition of SE SN templates from Modjaz et al. (2014). For illustration, the classification spectra for nine $\mathrm{SNe}$ of the sample are shown in Fig. 1. The SNe have been selected in order to illustrate the variety of data quality of the spectral sample. We also note that that details provided by SNID, such as phases and redshifts are in agreement with the actual value for each $\mathrm{SN}$ within the uncertainties.

The objects classified as Type Ibc here are those for which a clear difference between Ib and Ic could not be established, due to the spectral-data quality. This represents the full sample of the PTF + iPTF (hereafter combined into (i)PTF) Type Ic population and our classifications are mostly consistent with those by Fremling et al. (2018). The redshift of the host galaxy has been adopted when available ${ }^{1}$. When this information was not available, the redshift was estimated from the host-galaxy lines when detected, otherwise we adopted the best fit from SNID. The redshifts of the sample span the interval $z=0.004486-0.176$. The mean value is $z=0.049 \pm 0.033$. The redshift distribution is presented in Fig. 2.

1 The values refer to the ones available at the NASA/IPAC extragalactic database; http://ned.ipac. caltech.edu 


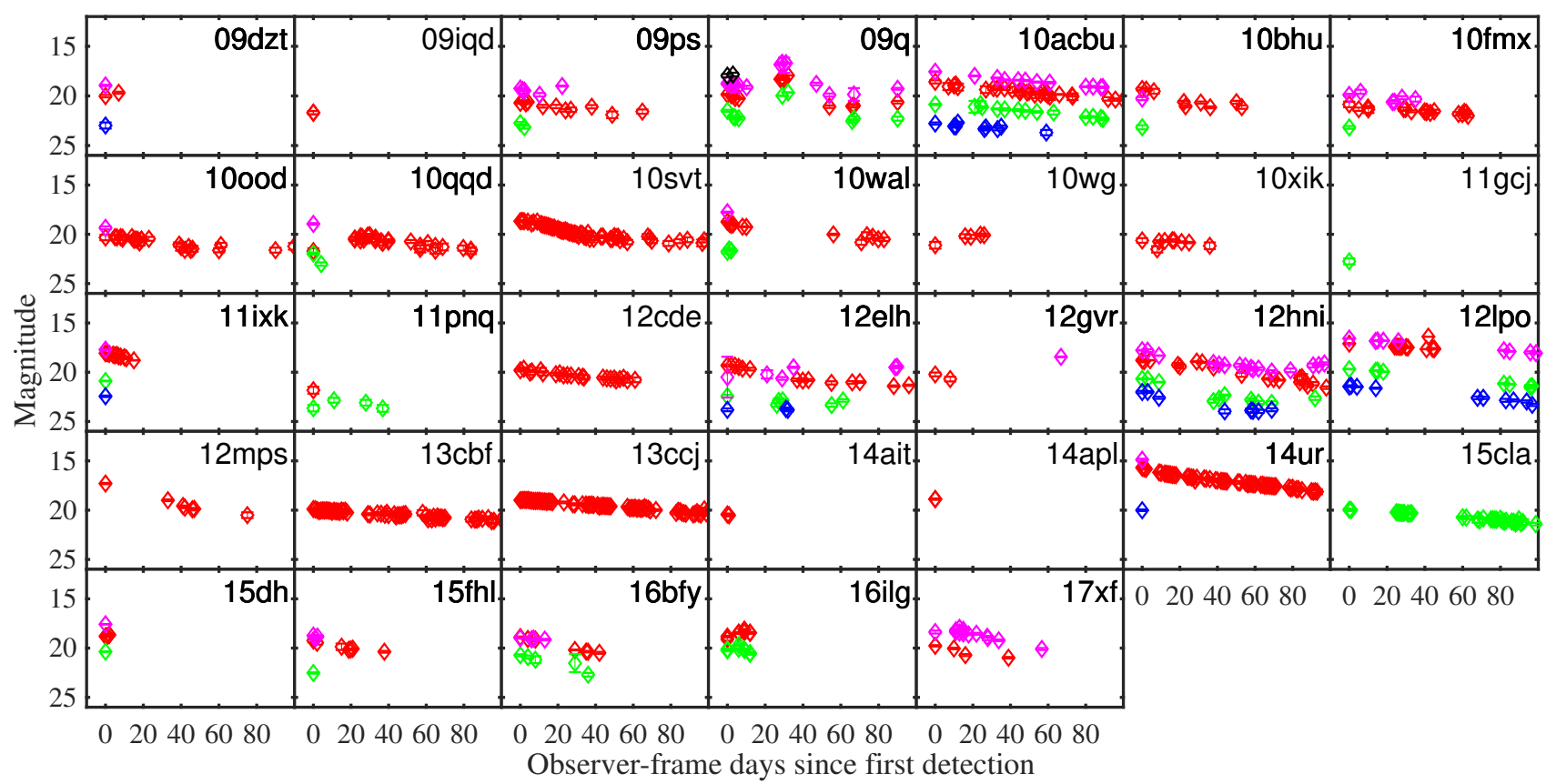

Fig. 3. Light curves for all the $33 \mathrm{SNe}$ Ic and Ibc in (i)PTF which do not show an observed peak and will not be included in the overall analysis. We plot the apparent magnitude as a function of time since first detection, in the observer's frame. Shifts have been applied for clarity as indicated in the legend in the bottom row.

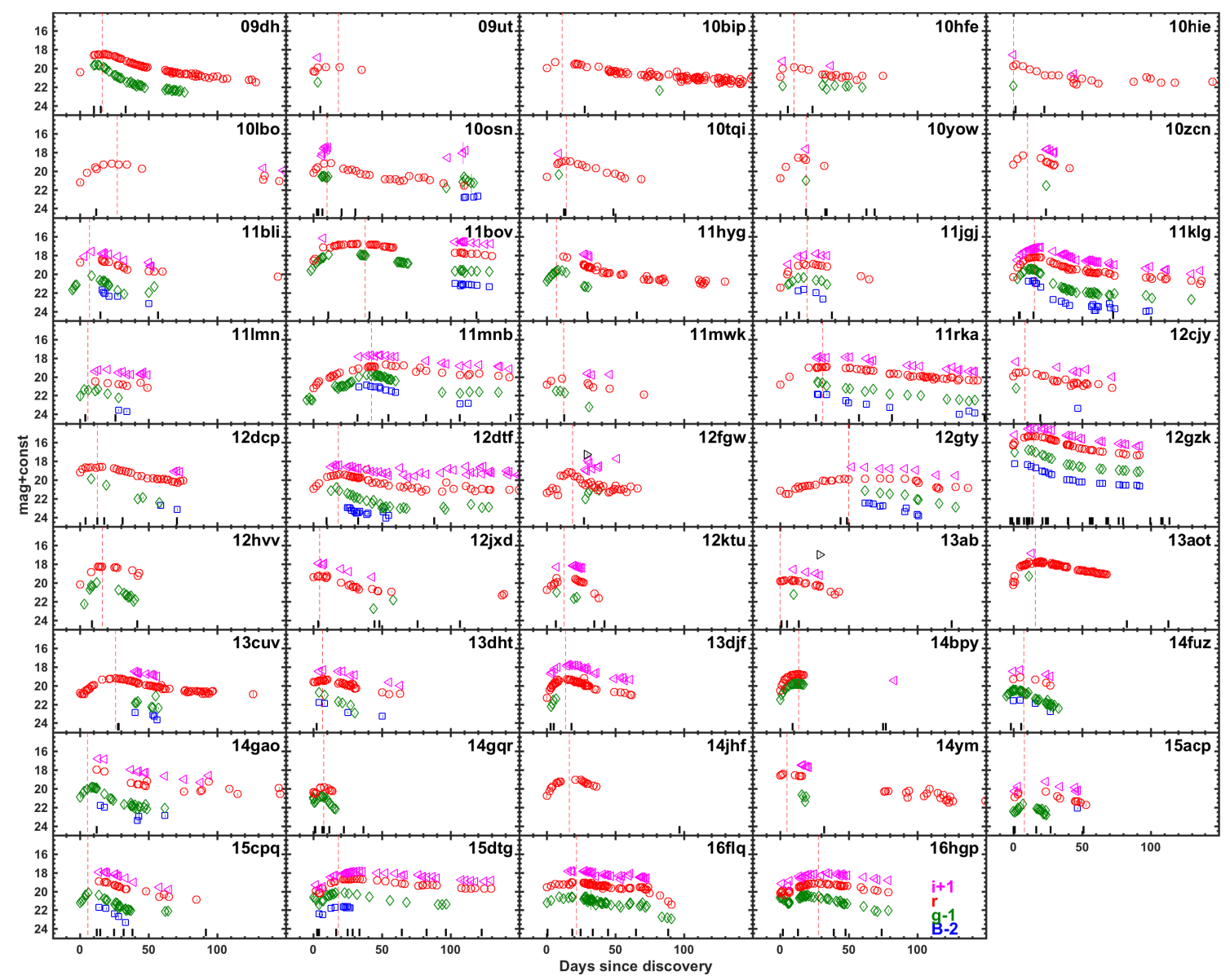

Fig. 4. Light curves in $B, g, r, i$ of the $44 \mathrm{SNe}$ Ic for which we have pre-maximum observations. We plot the apparent magnitude as a function of days since discovery. Shifts have been applied for clarity, as indicated in the legend in the bottom row. The peak epoch is shown as a dashed red line. The black dashed lines at the bottom represent epochs of spectral observations. 
Table 1. 44 SNe Ic in the sample used in this work with their coordinates, redshifts, distances and extinction estimates.

\begin{tabular}{|c|c|c|c|c|c|c|c|c|c|c|}
\hline $\mathrm{SN}$ & Type & $\begin{array}{c}\text { RA } \\
\text { (h m s) }\end{array}$ & $\begin{array}{l}\text { Dec } \\
\left({ }^{\circ \prime \prime \prime}\right)\end{array}$ & $z$ & $\begin{array}{c}\text { Distance modulus } \\
\text { (mag) }\end{array}$ & $\begin{array}{c}\text { Distance } \\
(\mathrm{Mpc})\end{array}$ & $\begin{array}{c}E(B-V)_{\mathrm{MW}} \\
(\mathrm{mag})\end{array}$ & $\begin{array}{l}E(B-V)_{\text {host }} \\
\text { from Na I D } \\
\qquad(\mathrm{mag})\end{array}$ & $\begin{array}{l}E(B-V)_{\text {host }} \\
\text { from Na I D } \\
\qquad(b) \\
(\mathrm{mag})\end{array}$ & $\begin{array}{l}E(B-V)_{\text {host }} \\
\text { from } g-r \\
\quad(\mathrm{mag})\end{array}$ \\
\hline 09dh & Ic & $14: 44: 42.07$ & $+49: 43: 44.9$ & $0.07^{(1)}$ & 37.48 & 313.91 & 0.0213 & 0.16 & $0.21(0.07)$ & $0.14(0.14)$ \\
\hline 09ut & $\mathrm{Ibc}$ & $14: 12: 07.64$ & $+74: 45: 46.0$ & $0.04^{(1)}$ & 36.33 & 184.50 & 0.0260 & 0.00 & $0.01(0.01)$ & $0.24(0.25)$ \\
\hline 10bip & Ic & $12: 34: 10.52$ & $+08: 21: 48.5$ & 0.051 & 36.77 & 225.55 & 0.0159 & 0.06 & $0.04(0.01)$ & $0.00(0.00)$ \\
\hline $10 \mathrm{hfe}$ & Ic & $12: 32: 05.16$ & $+66: 24: 23.9$ & 0.049 & 36.68 & 216.38 & 0.0157 & 0.00 & $0.01(0.01)$ & $0.00(0.00)$ \\
\hline 10hie & Ic & $17: 02: 27.71$ & $+28: 31: 57.6$ & $0.067^{(2)}$ & 37.38 & 299.81 & 0.0464 & 0.02 & $0.02(0.01)$ & $0.33(0.25)$ \\
\hline 10lbo & Ic & $12: 59: 14.79$ & $+61: 27: 02.2$ & $0.053^{(2)}$ & 36.85 & 234.74 & 0.0138 & 0.00 & $0.01(0.01)$ & $0.00(0.00)$ \\
\hline $10 \mathrm{osn}$ & Ic & $23: 23: 07.98$ & $+17: 30: 29.1$ & 0.038 & 36.11 & 166.43 & 0.0361 & 0.00 & $0.01(0.01)$ & $0.06(0.12)$ \\
\hline 10tqi & Ic & $23: 20: 47.73$ & $+18: 54: 17.3$ & 0.038 & 36.14 & 169.13 & 0.0347 & 0.16 & $0.21(0.07)$ & $0.18(0.25)$ \\
\hline 10yow & Ic & $21: 54: 23.30$ & $+15: 09: 20.7$ & 0.024 & 35.13 & 106.34 & 0.0902 & 0.18 & $0.27(0.10)$ & $0.22(0.25)$ \\
\hline $10 \mathrm{zcn}$ & Ic & $23: 19: 14.39$ & $+26: 03: 11.6$ & $0.02^{(1)}$ & 34.78 & 90.25 & 0.0794 & 0.12 & $0.10(0.04)$ & $0.64(0.25)$ \\
\hline 11bli & Ibc & $14: 02: 16.18$ & $+33: 39: 41.5$ & 0.034 & 35.88 & 149.80 & 0.0124 & 0.10 & $0.07(0.03)$ & $0.49(0.16)$ \\
\hline $11 \mathrm{bov}$ & Ic & $12: 56: 53.94$ & $+22: 22: 28.1$ & 0.021 & 34.90 & 95.62 & 0.0289 & 0.05 & $0.03(0.01)$ & $0.00(0.00)$ \\
\hline 11hyg & Ic & $23: 27: 57.34$ & $+08: 46: 38.0$ & 0.029 & 35.50 & 125.81 & 0.0509 & 0.10 & $0.08(0.03)$ & $0.26(0.14)$ \\
\hline $11 \mathrm{jgj}$ & Ic & $16: 31: 32.35$ & $+62: 06: 09.5$ & $0.040^{(2)}$ & 36.22 & 175.45 & 0.0257 & 0.22 & $0.61(0.22)$ & $0.26(0.12)$ \\
\hline $11 \mathrm{klg}$ & Ic & 22:07:09.92 & $+06: 29: 08.7$ & 0.026 & 35.31 & 115.15 & 0.0754 & 0.00 & $0.01(0.01)$ & $0.28(0.18)$ \\
\hline $11 \mathrm{lmn}$ & $\mathrm{Ibc}$ & $17: 30: 16.33$ & $+26: 59: 34.0$ & $0.090^{(2)}$ & 38.07 & 411.33 & 0.0439 & 0.00 & $0.01(0.01)$ & $0.00(0.00)$ \\
\hline $11 \mathrm{mnb}$ & Ic & $00: 34: 13.25$ & $+02: 48: 31.4$ & $0.060^{(2)}$ & 37.14 & 268.51 & 0.0157 & 0.08 & $0.05(0.02)$ & $0.08(0.17)$ \\
\hline $11 \mathrm{mwk}$ & $\mathrm{Ibc}$ & $21: 35: 01.39$ & $+00: 07: 16.0$ & $0.121^{(2)}$ & 38.76 & 564.78 & 0.0457 & 0.02 & $0.02(0.01)$ & $0.00(0.00)$ \\
\hline 11rka & Ic & $12: 40: 44.87$ & $+12: 53: 21.4$ & $0.074^{(2)}$ & 37.62 & 334.70 & 0.0295 & 0.08 & $0.05(0.02)$ & $0.29(0.13)$ \\
\hline $12 \mathrm{dtf}$ & Ic & $17: 14: 43.72$ & $+45: 18: 19.0$ & $0.061^{(2)}$ & 36.43 & 193.58 & 0.0347 & 0.00 & $0.01(0.01)$ & $0.36(0.16)$ \\
\hline $12 \mathrm{dcp}$ & Ic & $16: 12: 56.12$ & $+32: 30: 43.2$ & 0.031 & 35.65 & 134.74 & 0.0204 & 0.00 & $0.01(0.01)$ & $0.38(0.17)$ \\
\hline 12 cjy & Ic & $14: 34: 27.31$ & $+51: 49: 03.7$ & 0.044 & 37.17 & 271.77 & 0.0082 & 0.04 & $0.03(0.01)$ & $0.00(0.00)$ \\
\hline $12 \mathrm{fgw}$ & Ic & $15: 53: 27.12$ & $+33: 21: 04.2$ & 0.055 & 36.94 & 243.96 & 0.0287 & 0.00 & $0.01(0.01)$ & $0.00(0.00)$ \\
\hline $12 \mathrm{gty}$ & Ic & $16: 01: 15.23$ & $+21: 23: 17.4$ & $0.17^{(1)}$ & 39.64 & 847.56 & 0.0589 & 0.03 & $0.02(0.01)$ & $0.04(0.16)$ \\
\hline $12 \mathrm{gzk}$ & Ic & $22: 12: 41.53$ & $+00: 30: 43.1$ & $0.013^{(2)}$ & 33.86 & 59.20 & 0.0432 & 0.14 & $0.14(0.05)$ & $0.21(0.12)$ \\
\hline $12 \mathrm{hvv}$ & Ic & $21: 45: 46.45$ & $-00: 03: 25.1$ & $0.029^{(2)}$ & 35.50 & 126.14 & 0.0715 & 0.08 & $0.05(0.02)$ & $0.70(0.22)$ \\
\hline $12 \mathrm{jxd}$ & Ic & 09:37:29.82 & $+23: 09: 50.2$ & 0.025 & 35.21 & 110.18 & 0.0258 & 0.32 & $0.30(1.14)$ & $0.43(0.25)$ \\
\hline $12 \mathrm{ktu}$ & Ic & $04: 26: 20.58$ & $-10: 06: 12.5$ & 0.031 & 35.65 & 135.05 & 0.0567 & 0.08 & $0.05(0.02)$ & $0.34(0.12)$ \\
\hline $13 \mathrm{ab}$ & Ic & $12: 38: 12.51$ & $+07: 09: 02.0$ & 0.048 & 36.63 & 211.81 & 0.0183 & 0.04 & $0.03(0.01)$ & $0.00(0.00)$ \\
\hline 13aot & Ic & $13: 18: 26.09$ & $+31: 28: 09.9$ & 0.018 & 34.53 & 80.65 & 0.0103 & 0.04 & $0.02(0.01)$ & - \\
\hline $13 \mathrm{cuv}$ & Ic & $01: 53: 20.32$ & $+35: 50: 19.3$ & $0.049^{(2)}$ & 36.48 & 198.13 & 0.0605 & 0.02 & $0.04(0.01)$ & $0.26(0.13)$ \\
\hline $13 \mathrm{dht}$ & Ic & $23: 44: 58.88$ & $+09: 55: 24.8$ & $0.040^{(2)}$ & 36.22 & 175.45 & 0.0658 & 0.06 & $0.02(0.01)$ & $0.20(0.17)$ \\
\hline 13djf & Ic & $23: 33: 38.73$ & $+08: 48: 44.6$ & 0.021 & 34.70 & 87.07 & 0.0690 & 0.03 & $0.01(0.01)$ & $0.57(0.25)$ \\
\hline 14bpy & Ic & $15: 33: 08.66$ & $+17: 17: 53.3$ & $0.04^{(1)}$ & 36.48 & 198.13 & 0.0361 & 0.00 & $0.03(0.01)$ & $0.00(0.00)$ \\
\hline 14gao & Ic & $00: 57: 40.21$ & $+43: 47: 35.2$ & 0.018 & 36.43 & 193.58 & 0.0688 & 0.04 & $0.00(0.01)$ & $0.55(0.14)$ \\
\hline $14 \mathrm{gqr}$ & Ic & $23: 33: 27.95$ & $+33: 38: 46.1$ & $0.063^{(2)}$ & 34.45 & 77.64 & 0.0800 & 0.41 & $0.01(0.01)$ & $0.00(0.00)$ \\
\hline $14 \mathrm{fuz}$ & $\mathrm{Ibc}$ & 01:05:30.18 & $+02: 51: 42.0$ & 0.044 & 37.24 & 281.09 & 0.0235 & 0.00 & $0.00(0.01)$ & $0.00(0.00)$ \\
\hline 14jhf & $\mathrm{Ibc}$ & 08:00:33.06 & $+18: 15: 35.9$ & $0.053^{(2)}$ & 36.90 & 239.35 & 0.0202 & 0.31 & $0.17(0.98)$ & - \\
\hline $14 y m$ & Ic & $17: 46: 40.45$ & $+58: 38: 11.1$ & 0.031 & 35.68 & 136.83 & 0.0400 & 0.15 & $0.12(0.06)$ & $0.22(0.16)$ \\
\hline $15 \mathrm{acp}$ & Ic & $14: 53: 56.34$ & $+59: 02: 51.4$ & $0.138^{(2)}$ & 39.06 & 648.56 & 0.0094 & 0.13 & $0.01(0.04)$ & $0.33(0.14)$ \\
\hline $15 \mathrm{cpq}$ & $\mathrm{Ibc}$ & $00: 13: 43.16$ & $+00: 09: 43.2$ & $0.066^{(2)}$ & 37.35 & 295.12 & 0.0238 & 0.10 & $0.07(0.03)$ & $0.16(0.14)$ \\
\hline $15 \mathrm{dtg}$ & Ic & $02: 30: 20.05$ & $+37: 14: 06.7$ & $0.052^{(2)}$ & 36.83 & 231.98 & 0.0549 & 0.00 & $0.01(0.01)$ & $0.11(0.12)$ \\
\hline $16 \mathrm{flq}$ & $\mathrm{Ibc}$ & $00: 28: 36.54$ & $-01: 33: 03.3$ & $0.06^{(1)}$ & 37.13 & 266.65 & 0.0190 & 0.24 & $0.74(0.27)$ & $0.23(0.12)$ \\
\hline 16hgp & Ic & $00: 12: 06.41$ & $+32: 11: 50.9$ & $0.079^{(2)}$ & 37.76 & 356.57 & 0.038 & 0.00 & $0.01(0.01)$ & $0.06(0.14)$ \\
\hline
\end{tabular}

Notes. ${ }^{(a)} E(B-V)$ estimate with Na ID using the Taubenberger et al. (2006) relation. Adopted uncertainties of $\Delta E(B-V)=02$ mag. ${ }^{(b)} E(B-V)$ estimate with Na ID using the Poznanski et al. (2012) relation. ${ }^{(1)}$ Redshift estimated from SNID. ${ }^{(2)}$ Redshift estimated from narrow host line.

The redshift was used to compute the luminosity distance for each SN. We adopted the WMAP 5-year (Komatsu et al. 2009) cosmological parameters $H_{0}=70.5 \mathrm{~km} \mathrm{~s}^{-1} \mathrm{Mpc}^{-1}$, $\Omega_{\mathrm{M}}=0.27, \Omega_{\Lambda}=0.73$ and corrections for peculiar motions (Virgo, GA, Shapley) are included using a function by Nyholm et al. (2020) based on Mould et al. (2000a,b) and the NASA/IPAC Extragalactic Database velocity routine. With these assumptions, the distance of the sample ranges in the interval $D=19.1-847.6 \mathrm{Mpc}$.
The Milky Way extinction was obtained from Schlafly \& Finkbeiner $(2011)^{2}$. The treatment of the host extinction is presented in Sect. 4.3. Our sample was observed mainly in the $r$ and $g$ bands, with some photometric data also in the $B, i$ and $z$ bands for some objects. All the light curves in apparent magnitudes for the $77 \mathrm{SNe}$ are shown in Figs. 3 and 4.

\footnotetext{
2 Via the NASA/IPAC infrared science archive; https://irsa.
} ipac.caltech.edu/applications/DUST/ 
Among the $60 \mathrm{SNe}$ Ic $+17 \mathrm{SNe}$ Ibc of our sample, 44 were observed before peak in at least one band. In the following analysis we will focus only on these latter 44 objects. These $\mathrm{SNe}$ were observed in the $r$ band with an average cadence of 3 days and they have been followed with a median coverage of 66 days post peak. The $44 \mathrm{SNe}$ in the sample have a redshift in the interval $z=0.01377-0.176$, the mean value being $z=0.051 \pm 0.032$. The redshifts for the SNe are highlighted in Fig. 2 and listed in Table 1 where it is specified if the redshift was obtained with SNID or measured from narrow emission lines. They are all provided with 3 decimals.

This paper is mainly focused on the photometric data of the sample. However, each SN has at least one spectrum obtained by the (i)PTF survey and collaborators. The analysis of these spectra was published in Fremling et al. (2018). This (i)PTF data set of $\mathrm{SNe}$ Ic is thus unique given its untargeted nature, its large size, its early coverage, its high cadence and multiband coverage. A detailed analysis of the host galaxies of many of these Type Ic $\mathrm{SNe}$, and of the hosts of the PTF sample of broad lined Type Ic $\mathrm{SNe}$ (SNe Ic-BL), was presented by Modjaz et al. (2020). We compared the classification reported in Modjaz et al. (2020) with the one we present in this work. We notice that PTF09ps and PTF10bip are presented as Ic/Ic-BL, while PTF11gcj is classified as Ic-BL. We consider these three as $\mathrm{SNe}$ Ic as our classification suggested. The analysis of the (i)PTF sample of SNe Ic-BL was published by Taddia et al. (2019b). Here we focus on the spectroscopically normal (as opposed to broad lined) SNe Ic. Some of the SNe in our sample have already been studied in other works. SNe PTF09dh, PTF11bli, PTF11jgj, PTF11klg, PTF11rka and PTF12gzk were presented in Prentice et al. (2016). In that work PTF09dh was classified as a SN Ic-BL, but we include it here since we have re-classified it as a spectroscopically-normal SN Ic. We also note that PTF10vgv, presented as a SN Ic in Prentice et al. (2016), is not included in this work since we re-classified it as a SN Ic-BL; therefore, PTF10vgv was presented within the sample of SNe Ic-BL from (i)PTF in Taddia et al. (2019b) and also in Corsi et al. (2012). PTF12gzk was presented and discussed in Ben-Ami et al. (2012); PTF11bov is also known as SN 2011bm and was studied by Valenti et al. (2012) and Taddia et al. (2016); iPTF12gty was presented in De Cia et al. (2018) and also discussed by Quimby et al. (2018) as a superluminous supernova of Type I (SLSN-I). However, our spectroscopic classification suggests some similarity with a SN Ic so we included it in this work. iPTF15dtg was presented and discussed in Taddia et al. (2016) and late-time data are shown in Taddia et al. (2019a). PTF11 mnb was presented in a separate paper as a SN Ic (Taddia et al. 2018a) but it is also discussed in Quimby et al. (2018) as a possible SLSN-I. We include PTF11mnb in this work since our spectroscopic classification agrees with that of a SN Ic by Taddia et al. (2018a). Finally, iPTF14gqr was presented in De et al. (2018).

We notice that some $\mathrm{SNe}$, spectroscopically classified as normal SNe Ic, show quite broad light curves compared to the bulk of the sample ${ }^{3}$. In order to identify these SNe, the SE SN template presented by Taddia et al. (2015) was used as a reference. The template was shifted and stretched to fit the SN light curve at maximum. The SNe that presented a stretch factor higher than 1.5 were considered as having a broad light curve. The method used and these $\mathrm{SNe}$ will be discussed in detail in a forthcoming paper (Karamehmetoglu et al., in prep.). Our sample also

\footnotetext{
3 PTF11mnb, PTF11rka, PTF12gty, iPTF15dtg, iPTF16flq and iPTF16hgp.
}
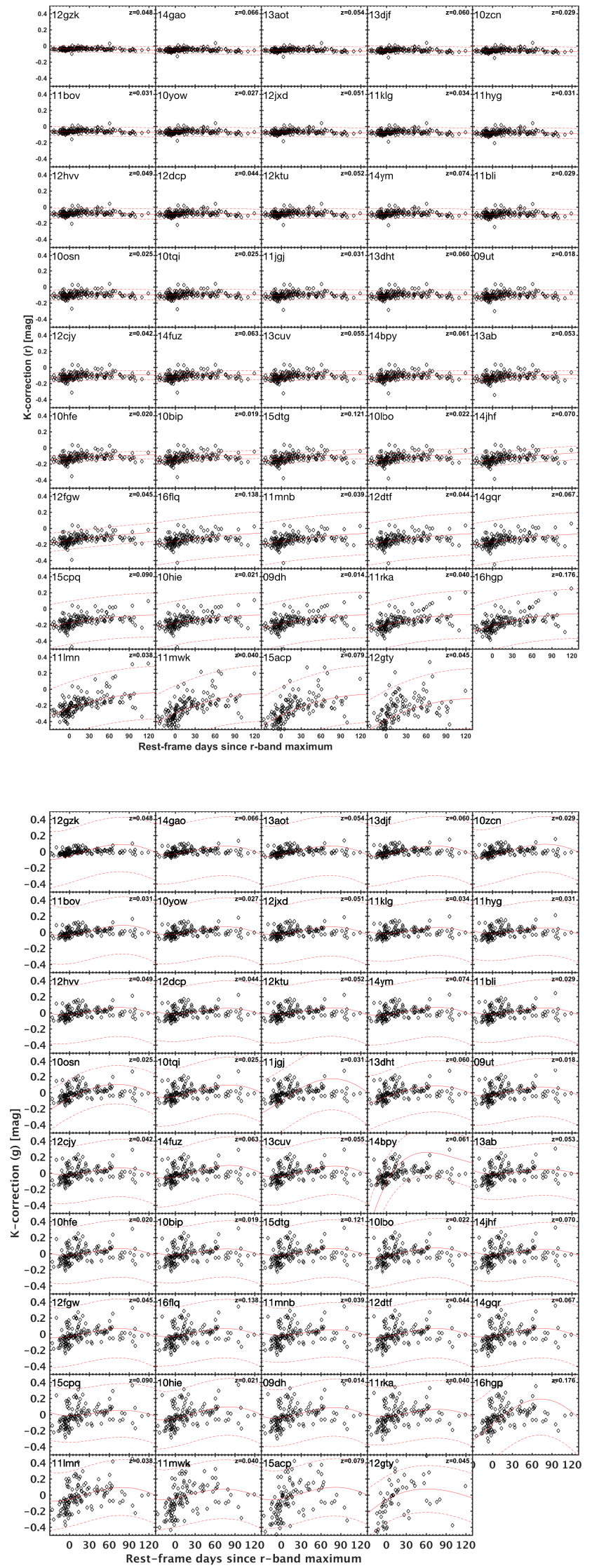

Fig. 5. K-corrections in the $r$ band for our SN sample. The solid red line represents the second order polynomial fit, whereas the red dashed lines show the $1 \sigma$ uncertainties. The $\mathrm{SNe}$ have been ordered according to increasing redshift. 

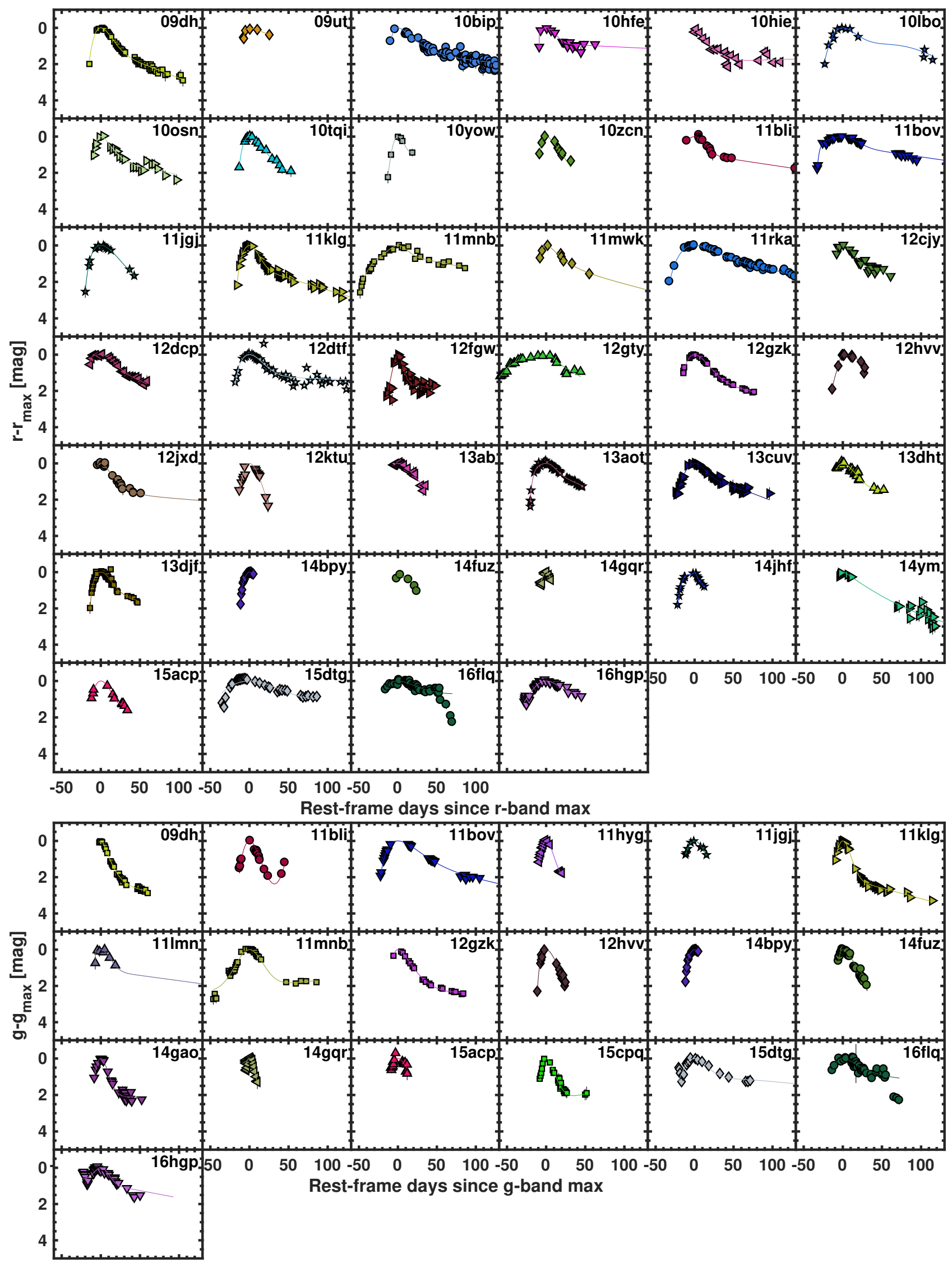

Fig. 6. Upper panel: individual $40 \mathrm{SNe}$ and their Contardo fits in the $r$ band. Lower panel: individual $19 \mathrm{SNe}$ and their Contardo fits in the $g$ band. 


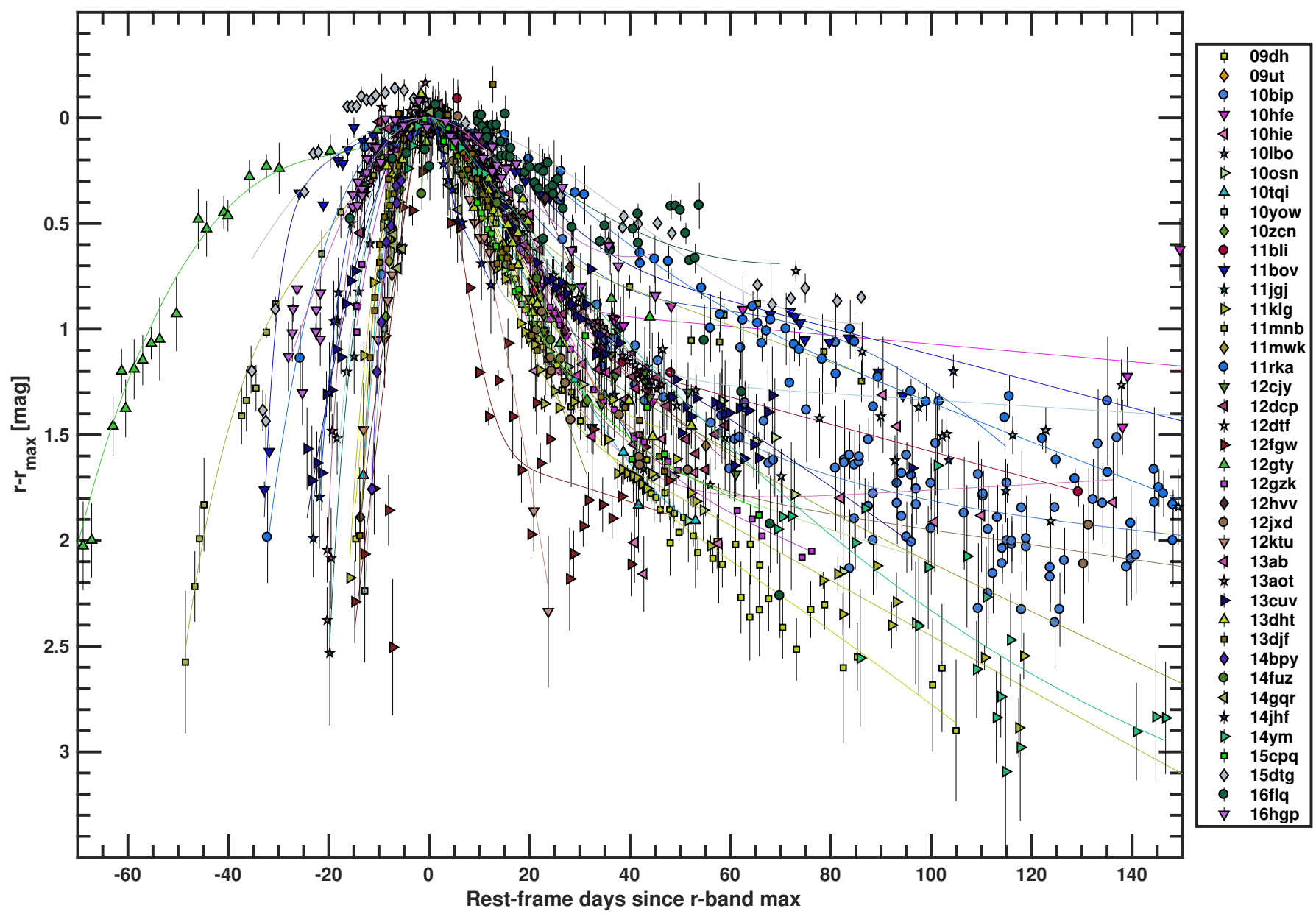

Fig. 7. $r$-band light curves for our $40 \mathrm{SNe}$ Ic plotted together. The best Contardo fits are included as full lines. In this and following plots the individual $\mathrm{SNe}$ are represented with symbols and colors as provided in the legend to the right. The light curves are normalized at peak in order to illustrate their diversity.

includes an object with a very narrow light curve ${ }^{4}$. The presence of a wider variety of of objects is likely due to large sample and the untargeted nature of the survey.

\section{Photometric observations and data reduction}

SN discovery and early photometric observations were performed with the 48-inch Samuel Oschin Telescope at Palomar Observatory (P48), equipped with the $96 \mathrm{Mpx}$ mosaic camera CFH12K (Rahmer et al. 2008), a Mould $r$-band filter (Ofek et al. 2012) and a $g$-band filter. For $34 \mathrm{SNe}$ in our sample, further follow-up was performed with the automated Palomar 60-inch telescope (P60, Cenko et al. 2006), often in Bgri bands. Point spread function (PSF) photometry was obtained on template subtracted images using the Palomar Transient Factory Image Differencing and Extraction (PTFIDE) pipeline (Masci et al. 2017) for P48 data and the FPipe pipeline presented in Fremling et al. (2016) for the P60 data. The photometry was calibrated using Sloan Digital Sky Survey (SDSS) stars (Ahn et al. 2014) in the $\mathrm{SN}$ field. All light curves will be released.

\section{Supernova light curves}

In Fig. 4 we present the photometric observations in the optical bands available for our SN sample. As mentioned before, we

\footnotetext{
4 iPTF14gqr.
}

will proceed with our analysis only for those $44 \mathrm{SNe}$ which have been observed before peak. The observed light curves were first corrected for time dilation and K-corrections.

\subsection{K-corrections}

Most of the SNe were observed in the $r$ band and we estimated the observed peak epoch in the $r$ band ( $t_{r}^{\max }$ ) by fitting a polynomial to the $r$-band light curves. The peak epoch is shown with a dashed red line in Fig. 4.

When the peak was observed also (15 objects) or only ( 4 objects) $)^{5}$ in the $g$ band, we performed the same fit in this band. The observed phases were corrected by a factor of $(1+z)$ to account for time dilation in order to obtain the phase in the rest frame. The measured $t_{r}^{\max }$ was used to determine the rest-frame phase of the SN spectra in our sample. The spectra were used to compute average K-corrections for the Bgri bands as a function of redshift and time since $t_{r}^{\max }$. The method we performed has been presented in Taddia et al. (2019b) and makes use of all the available spectra of the sample to estimate the K-corrections for every single SN. The main reason to apply this method is the lack of a complete spectral follow-up for most of the SNe of the

5 This was done for 19 SNe namely PTF09dh, PTF11bli, PTF11bov, PTF11hyg, PTF11jgj, PTF11klg, PTF11lmn, PTF11mnb, iPTF12gzk, iPTF12hvv, iPTF14bpy, iPTF14fuz, iPTF14gao, iPTF14gqr, iPTF 15acp, iPTF15cpq, iPTF15dtg, iPTF16flq and iPTF16hgp. 
Table 2. Parameters for the analysis of the lightcurve shape in the $r$ band for the $40 \mathrm{SNe}$.

\begin{tabular}{|c|c|c|c|c|c|}
\hline SN & $\begin{array}{l}t_{r}^{\max } \\
(\mathrm{JD}) \\
\end{array}$ & $\begin{array}{l}m_{r}^{\text {peak }} \\
(\mathrm{mag})\end{array}$ & $\begin{array}{c}\Delta m_{15}(r) \\
(\mathrm{mag})\end{array}$ & $\begin{array}{c}\Delta m_{-10}(r) \\
(\mathrm{mag})\end{array}$ & $\begin{array}{c}\Delta m_{40}(r) \\
(\mathrm{mag})\end{array}$ \\
\hline 09dh & 2454954.43(0.11) & $18.65(0.01)$ & $0.62(0.02)$ & $0.71(0.08)$ & $1.70(0.0$ \\
\hline 09ut & & $19.91(0.11)$ & $0.20(0.05)$ & - & \\
\hline 10bip & $2455231.72(0.12)$ & $19.38(0.05)$ & $0.43(0.01)$ & - & $1.17(0$. \\
\hline $10 \mathrm{hfe}$ & $2455341.19(1.41)$ & $20.00(0.11)$ & $0.50(0.03)$ & - & $0.94(0.11)$ \\
\hline 10hie & $2455336.27(0.01)$ & 19.71(0.08) & $0.73(0.05)$ & - & $1.62(0.13)$ \\
\hline 10lbo & 2 & 19.37 & $0.29(0$ & $0.26(0$. & 0.01 \\
\hline $10 \mathrm{osn}$ & & 19.27 & $0.65(0.01)$ & - & $151(008)$ \\
\hline 10tqi & 2455443. & 19.05 & $0.55(0.02)$ & $0.80(0.04)$ & $1.64(0.14)$ \\
\hline 10yow & 2455480.7 & $18.61(0.12)$ & $0.84(0.05)$ & $1.29(0.17)$ & 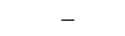 \\
\hline $10 \mathrm{zcn}$ & 245 & 18 & 0.65 & - & 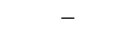 \\
\hline $11 \mathrm{bli}$ & 2455646 . & 18.61 & 0.49 ( & - & $.18(0$ \\
\hline $11 \mathrm{bov}$ & 2455685 & $16.85(0$ & $0.19(0.01)$ & $0.07(0.00)$ & $0.70(0.05)$ \\
\hline 11 hyg & $2455752.40(0.15)^{(a)}$ & - & - & - & - \\
\hline $11 \mathrm{jgj}$ & & 19.03(0.05) & $0.37(0.08)$ & $0.34(0.04)$ & $1.52(0.16)$ \\
\hline $11 \mathrm{k}$ & & 18.25 & 0.76 & 0.62 & 1.66 \\
\hline $111 \mathrm{mn}$ & 2455813.4 & - & e & 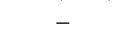 & ${ }^{2}$ \\
\hline $11 \mathrm{mnb}$ & & 2) & 0 . & 7) & 0.8 \\
\hline $11 \mathrm{mwk}$ & 2455829.79 & $20.48(0.19)$ & $0.48(0.05)$ & - & $1.24(0.04)$ \\
\hline 11rka & & 19.11 & 0.14 & $0.11(0.01)$ & $0.60(0.05)$ \\
\hline $12 \mathrm{cj}$ & & 19.59 & 0.53 & - & 1.31 \\
\hline $12 \mathrm{dcp}$ & & 18.74 & 0.32 & $0.07(0.03)$ & 1.22 \\
\hline 12 & & & 0.41 & & 1.12 \\
\hline $12 \mathrm{fgw}$ & 2 & $19.30(\mathrm{C}-\mathrm{C}) \mathrm{r}-\mathrm{x}$ & 1.52( & $1.45(\mathrm{C}$ & $1.82(0.05)$ \\
\hline 12 & & & 0.4 & 0.11 & 1.11 \\
\hline $12 \mathrm{~g}$ & & 15.37 & 0.46 & 0.42( & $1.49(0.01)$ \\
\hline $12 \mathrm{hv}$ & & 18.34 & 0.14 & $1.21(0.07)$ & 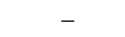 \\
\hline $12 \mathrm{jxd}$ & 2456229.9 & $19.33(0.07)$ & $0.67(0.03)$ & - & $54(0.04)$ \\
\hline $12 \mathrm{ktu}$ & & $19.37(0.03)$ & $1.06(0.14)$ & $9(0.13)$ & - \\
\hline & & & & - & - \\
\hline $13 \mathrm{a}$ & 20 & 17.88( & $0.43(($ & $0.16(0.01)$ & $1.11(0.01)$ \\
\hline $13 \mathrm{cu}$ & $56537.31(0.29)$ & $19.34(0$ & $0.43(0.04)$ & $0.32(0.01)$ & $1.13(0.06)$ \\
\hline $13 \mathrm{dht}$ & $2456551.36(0.89)$ & $19.49(0.07)$ & $0.51(0.04)$ & - & $1.41(0.11)$ \\
\hline 13djf & & $19.40(0$. & $0.60(0.04)$ & $0.65(0.14)$ & $1.46(0.09)$ \\
\hline 14bpy & & $1889(0)-2$ & - & $1.05(0.14)$ & - \\
\hline $14 f u z$ & & $19.05(0.04)$ & $0.43(0.05)$ & - & - \\
\hline 14gao & $2456925.59(0.83)^{(a)}$ & - & - & - & - \\
\hline $14 \mathrm{gqr}$ & $2456951.88(0.05)$ & $20.02(0.04)$ & - & - & - \\
\hline 14jhf & & & - & $0.16(0.02)$ & - \\
\hline $14 \mathrm{y}_{1}$ & & $18.49(0$ & $0.38(0$. & - & $7(0$. \\
\hline $15 \mathrm{a}$ & 245712224026 & $20.33(0.08)$ & $0.75(0.04)$ & $0.64(0.03)$ & - \\
\hline $15 \mathrm{cpq}$ & $2457283.61(0.40)^{(a)}$ & - & - & - & - \\
\hline $15 \mathrm{dtg}$ & $2457372.83(0.06)$ & $18.93(0.01)$ & $0.09(0.02)$ & $0.05(0.02)$ & $0.47(0.01)$ \\
\hline $16 \mathrm{fl}$ & & 07) & 0.16( & 0.17 & $0.52(0$ \\
\hline 16hgp & $2457712.03(0.07)$ & $19.36(0.04)$ & $0.25(0.01)$ & $0.16(0.05)$ & $0.66(0.07)$ \\
\hline
\end{tabular}

Notes. Uncertainties are in parenthesis. ${ }^{(a)}$ Epoch of $r$-band peak estimated from the $g$-band peak, considering the average shift estimated in Sect. 4.2.

sample. All the obtained K-corrections were then plotted as a function of the phase and fitted with a second-order polynomial. These fits in the $r$ band are shown in Fig. 5 .

Overall, we can see that the K-corrections are negligible for most of the objects but when present, at higher $z$, they are more important at early epochs. We K-corrected all the $g$ - and $r$-band light curves interpolating the above mentioned polynomials at all epochs of the light curve observations. In the following analysis, we will always refer to our K-corrected and time-dilation corrected light curves.

\subsection{Light curve shape}

We fitted the K-corrected $r$ - and $g$-band light curves with the function provided by Contardo et al. (2000) to characterize their
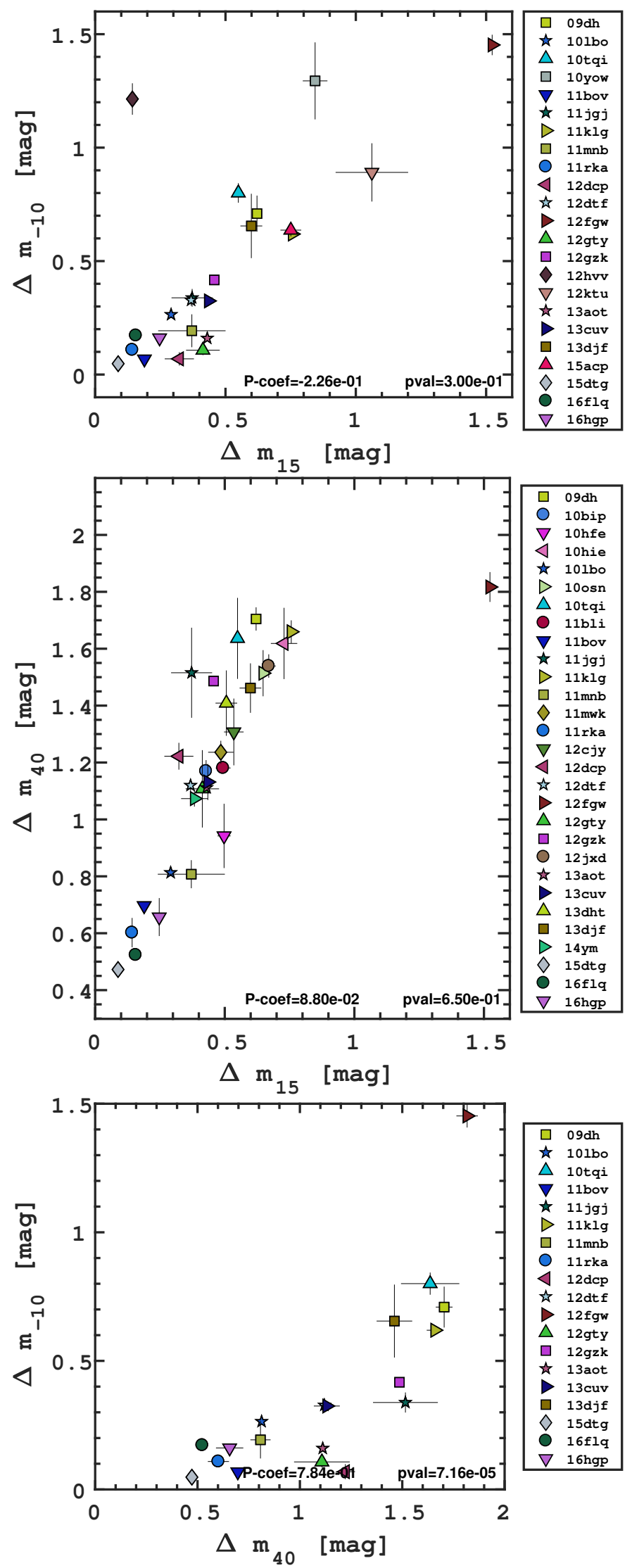

Fig. 8. Correlations between rise and decay in the $r$ band. Upper panel: $\Delta m_{15}$ against $\Delta m_{-10}$. Mid panel: $\Delta m_{15}$ vs. $\Delta m_{40}$. Lower panel: $\Delta m_{40}$ against $\Delta m_{-10}$. Plots show a correlation among parameters and their $p$ values, along with the Pearson coefficients are also reported. 

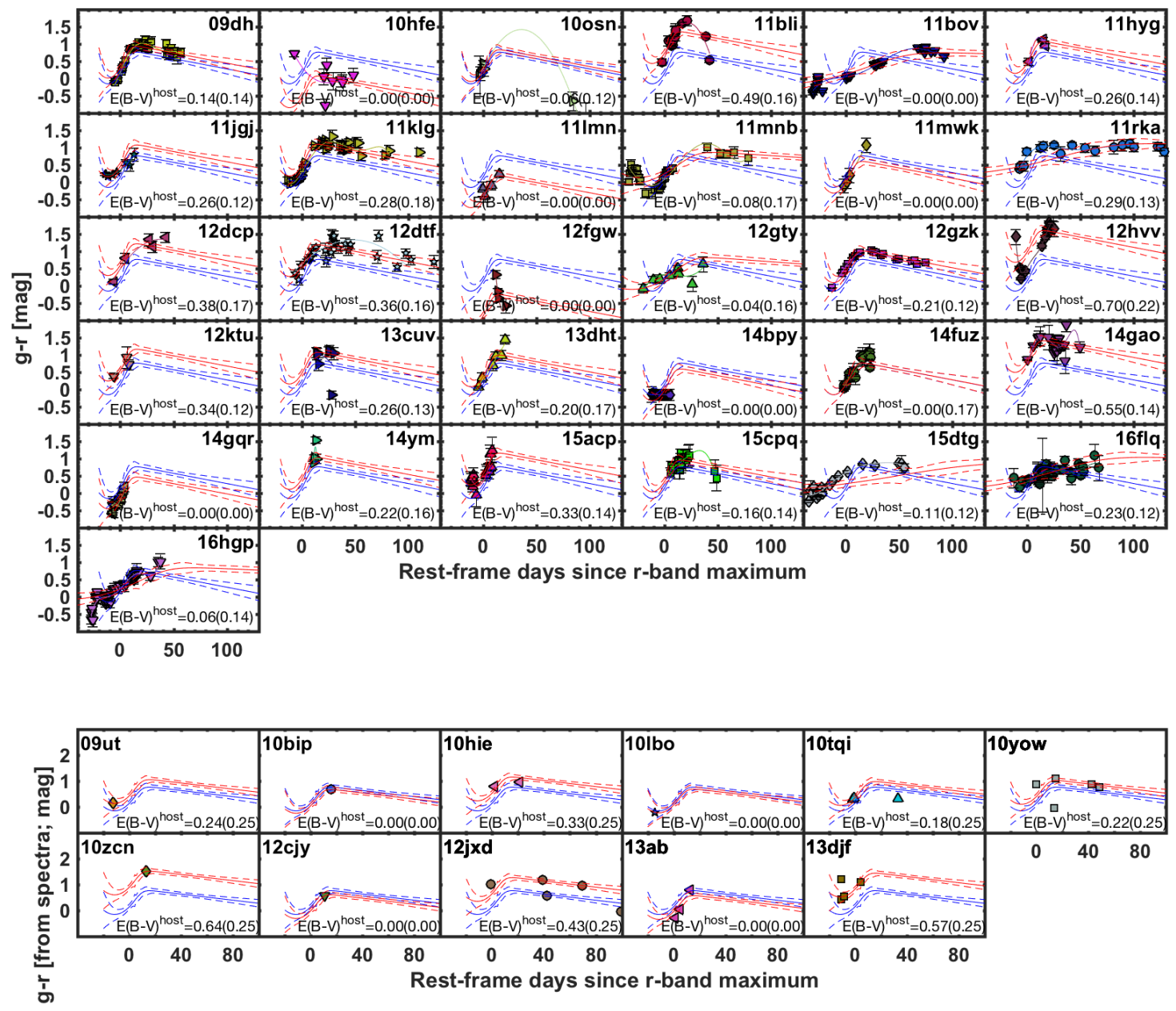

Fig. 9. Upper panel: individual (MW corrected) $g-r$ color evolution for $31 \mathrm{SNe}$ from photometry with the polynomial fits represented as solid lines. The red lines represent the fit of the data with the $g-r$ template. The blue line is the template for a Type Ic with no extinction from Stritzinger et al. (2018), and each panel shows the reddening in $E(B-V)$ required to shift the color curve to the data. This represents the estimated host extinction measured in magnitudes. Bottom panel: same as above for the $11 \mathrm{SNe}$ where the colors are calculated from spectroscopy.

shapes. This function includes an exponential rise, two Gaussian peaks, and a linear late decline. We included only the first of the two Gaussian peaks in our fit. From this fit it is possible to derive the peak epoch and magnitude, the rise parameter $\Delta m_{-10}$ as well as the decline parameters $\Delta m_{15}, \Delta m_{40}$ and the late linear decline slope. The parameter $\Delta m_{-10}$ measures how many magnitudes the light curve rises during the 10 rest frame days before peak. $\Delta m_{15}$ instead represents the decrease in magnitude 15 days after peak and $\Delta m_{40}$ at 40 days after peak. The results of our fits to the $r$ and $g$-band light curves are shown in Fig. 6.

In the top panel, each $\mathrm{SN}$ is represented individually in the $r$ band while the bottom panel shows the SNe in the $g$ band.

We focus our analysis on the shape of the light curves in the $r$ band. This leads to the exclusion of PTF11hyg, PTF11lmn, iPTF14gao and iPTF15cpq from our analysis since they only show the peak in the $g$ band. In Fig. 7 we present all our 40 $\mathrm{SNe}$ together to show the general shape of their light curves in the $r$ band.

This highlights the variety of rise and decline rates of the $\mathrm{SNe}$ of our sample. Through a Monte-Carlo procedure and simulating $N=100$ light curves, we estimate the uncertainties on each of the light curve fit parameters according to their photometric uncertainties. The uncertainty on each parameter is represented by the standard deviations of the best fit parameters.
These parameters and their estimated uncertainties are reported in Table 2.

Figure 8 suggests a correlation between $\Delta m_{15}$ and $\Delta m_{-10}$, with fast rising SNe also being fast declining. We also show the relation between $\Delta m_{15}$ and $\Delta m_{40}$ and the one between $\Delta m_{-10}$ and $\Delta m_{40}$ which also seem to suggest a correlation among these parameters. The $\Delta m_{-10}$ vs. $\Delta m_{40}$ gives a similar relation as $\Delta m_{-10}$ vs. $\Delta m_{15}$, implying that the fast rising $\mathrm{SNe}$ are the fast declining $\mathrm{SNe}$ also at 40 days past peak. The $\Delta m_{15}$ vs. $\Delta m_{40}$ demonstrates that the SNe that decline fast during the first 2 weeks are also the ones that declined more rapidly at later phases. We performed a Pearson test to quantify these correlations and the $p$-values are reported in Fig. 8. This test will be performed for all correlations in this work and $p$-values will be displayed in the plots. The $\Delta m_{40}$ and $\Delta m_{-10}$ show a lower p-value than the other two which indicates a stronger correlation, this is most likely due to the presence of an outlier in the other correlations. We estimated the epoch of the peak in $g$ band for $19 \mathrm{SNe}$ of the sample and compared it with the same estimate for the $r$ band. We find on average a shift between the peaks at $g$ and $r$ band of $4 \pm 2$ days, with the SN peaking in the $g$ band first. This is consistent with the estimate of $\sim 3$ days presented in Taddia et al. (2015). Armed with the average time shift between peaks in $g$ and $r$ band we can provide an estimate of the $r$-band peak for the 
$4 \mathrm{SNe}$ which show a peak only in the $g$ band. These values are also presented in Table 2 .

\subsection{Colours and host extinction}

We proceed to compute the color evolution of the $\mathrm{SNe}$, see Fig. 9.

For $31 \mathrm{SNe}$ with $g$ - and $r$-band data available, we corrected for the Milky Way (MW) extinction adopting the MW $E(B-V)$ given in Table 1 , assuming $R_{V}=3.1$ and a Fitzpatrick (1999) reddening law. We then interpolated the $r$ band to the $g$-band epochs to obtain the $g-r$ evolution. The color evolution tends to show an initial rise until approximately 20 days after peak, then it starts a shallow decline (getting bluer) at later epochs (Fig. 9). We estimated the host extinction from spectroscopy from the measurement of the equivalent width of the narrow Na ID absorption line. We followed Taubenberger et al. (2006, their Eq. (1)) to get $E(B-V)$ and adopt an uncertainty of $\Delta E(B-V)=0.2$ mag.

We also estimated $E(B-V)$ using Poznanski et al. (2012, their Eq. (9)) and notice that the values we get are in agreement with the previous estimates. The average difference between the two methods is $0.05 \pm 0.11$. We note that for PTF11jgj and iPTF16flq we find the biggest difference for the extinction estimates from $\mathrm{Na} I \mathrm{D}$ which also differ significantly from the estimates we get from the $g-r$ method. If we exclude these two $\mathrm{SNe}$ from the comparison we get that the average difference between the two methods is $0.03 \pm 0.06$. Since the difference is rather small, we will adopt the $E(B-V)$ from Taubenberger et al. (2006) when referring to extinction from $\mathrm{Na} I \mathrm{D}$ through the manuscript.

In addition, we used the $\mathrm{SN} g-r$ colors to estimate the host extinction, following the method described by Stritzinger et al. (2018). We fit the $g-r$ colors of all the SNe with low-order polynomials, shown as solid lines in Fig. 9. We then estimated the average $E(g-r)$ for each SN in the range between 0 and 20 days after the peak by computing the average difference between the fit of the observed $g-r$ and the assumed intrinsic $g-r$ color. We adopted the $g-r$ template presented in Stritzinger et al. (2018).

The $6 \mathrm{SNe}$ that present broader light curves as mentioned in Sect. 2 require a stretching factor to apply to our $g-r$ template in order to get a good fit (see Karamehmetoglu et al., in prep.). We estimated the stretch factor from the ratio between the average $\Delta m_{15}$ of the sample and the $\Delta m_{15}$ estimated for the individual SN with broad light curve, and then set the time scale from the ratio of the epochs of the peak in the $g-r$ color evolution of one broad SN (iPTF16hgp) and one normal (PTF12gzk). We then converted $E(g-r)$ into $E(B-V)_{\text {host }}$, assuming $R_{V}=3.1$. The uncertainty of the $g-r$ template is included in the uncertainties of the host galaxy extinctions. The uncertainty also takes into account the standard deviation due to the difference between the epochs of the measured color and the intrinsic $g-r$. We also used the spectra corrected for redshift and Milky Way extinction to build $g-r$ color curves for some $\mathrm{SNe}^{6}$ for which it was not possible to use the photometry (see bottom panel of Fig. 9). The computed $E(B-V)_{\text {host }}$ values are reported in Table 1 . We note that for two $\mathrm{SNe}^{7}$ it was not possible to get a $g-r$ evolution.

When we compare the host galaxy extinction estimates obtained from the SN color comparison to that obtained from

\footnotetext{
6 In particular, spectra were used for $11 \mathrm{SNe}$, namely PTF09ut, PTF10bip, PTF10hie, PTF10lbo, PTF10tqi, PTF10yow, PTF10zcn, PTF12cjy, PTF12jxd, iPTF13ab and iPTF13djf.

7 This has been adopted for SNe iPTF13aot and iPTF14jhf.
}

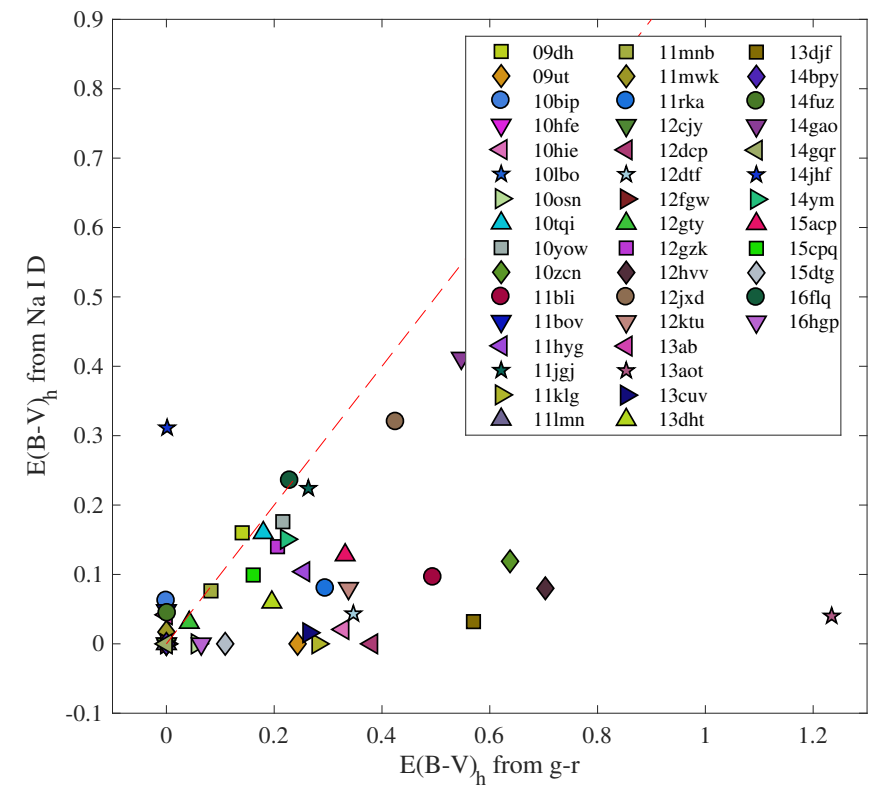

Fig. 10. A comparison between the extinction estimated from the $\mathrm{NaID}$ absorption vs. that estimated from the $g-r$ color evolution. The red line represents a one-to-one relation, but clearly the $\mathrm{Na} I \mathrm{D}$ gives consistently lower estimates. Units are in magnitudes.

the Na I D absorption lines, we notice that the first seems to provide overall higher values (Fig. 10).

Both of these methods come with considerable uncertainties and assumptions. We note that some of our spectra do not have enough signal-to-noise (S/N) close to the Na I D line to properly detect it. On the other hand, the $g-r$ method relies on an intrinsic color curve template and on assuming homogeneity of the color evolution for these SNe. In this work we will adopt the extinction estimated from the NaID, unless otherwise specified. The main reason is that we want to compare our results with those published in the literature, which most often have used this method. However, throughout the analysis we will discuss how some values will be affected if we instead chose the extinction estimated from the second method.

\subsection{Absolute magnitudes}

We applied the presented corrections; Milky Way and host extinctions, distances and K-corrections, to the light curves to obtain the absolute magnitudes, see Fig. 11.

The uncertainty on the absolute magnitudes takes into account the uncertainties due to the host extinction estimates and the photometric errors. In addition, the uncertainty on the distance adds a systematic error of \pm 0.15 mag which has not been included in the figure. This systematic is for an adopted uncertainty on $H_{0}$ of $\pm 5 \mathrm{~km} \mathrm{~s}^{-1}$, which dominates uncertainties from the peculiar velocities at the redshifts of our sample SNe. The distribution of the $r$-band absolute magnitudes at peak is shown in Fig. 12.

Our $r$-band magnitudes span the interval -15.45 to -19.73 mag when the host extinction has not been accounted for, giving an average of $\left\langle M_{r}^{\text {peak }}\right\rangle=-17.50 \pm 0.82 \mathrm{mag}$. It ranges from -15.54 to $-19.81 \mathrm{mag}$ when the host extinction from $\mathrm{Na}$ ID is included, with an average of $\left\langle M_{r}^{\text {peak }}\right\rangle=-17.71 \pm 0.85$ mag. If we instead consider the extinction estimates from $g-r$, the interval is -16.91 to $-19.84 \mathrm{mag}$ and an average of $\left\langle M_{r}^{\text {peak }}\right\rangle=$ 

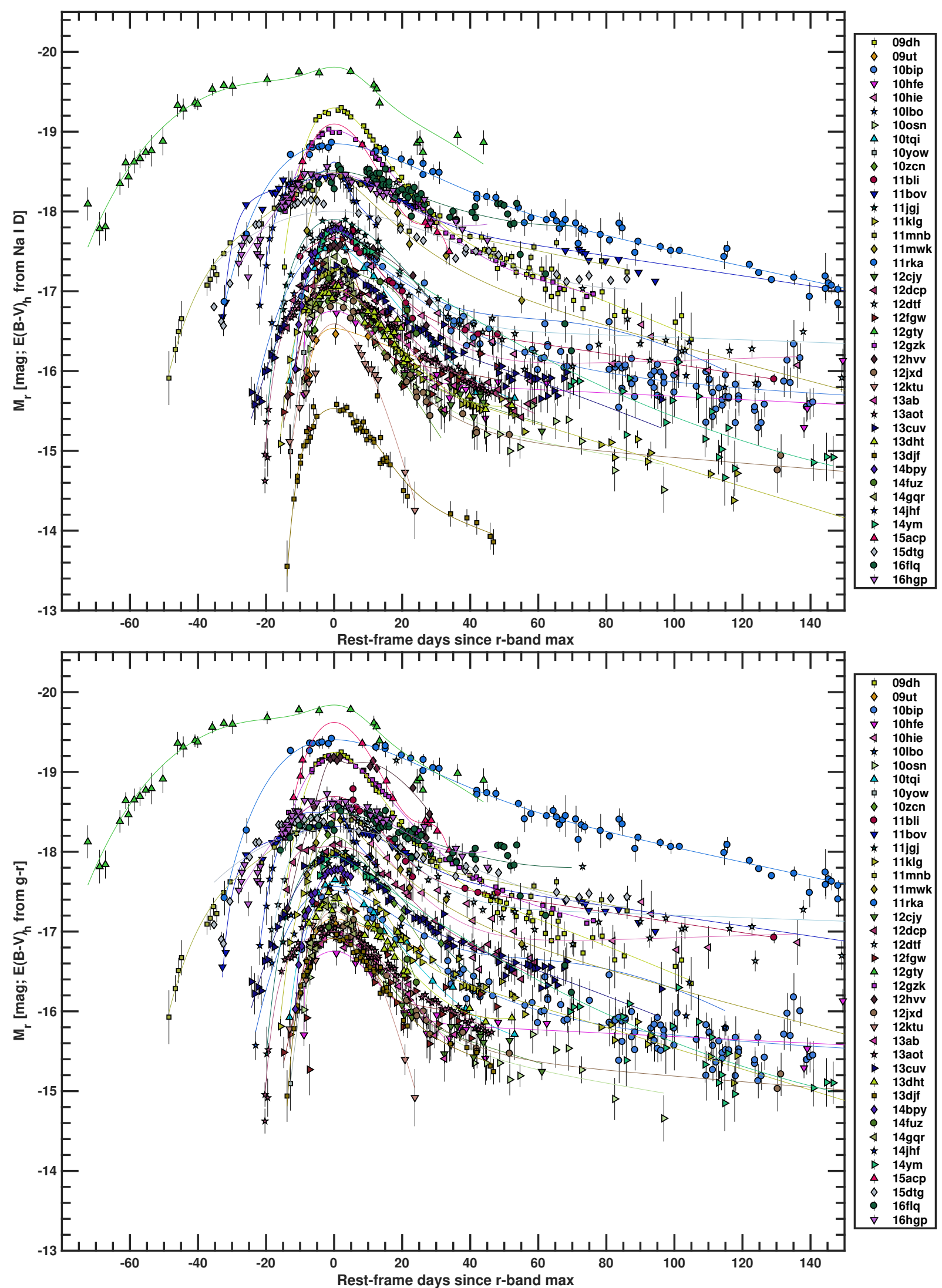

Fig. 11. Upper panel: absolute magnitude in $r$ band of the $40 \mathrm{SNe}$ of the sample when extinction is estimated from the Na ID absorption. Bottom panel: absolute magnitude in $r$ band of the $40 \mathrm{SNe}$ of the sample when extinction is estimated from $g-r$ color evolution. For SNe iPTF13aot and iPTF14jhf we assumed the extinction from the NaID in both cases, since there is no estimate from $g-r$. 


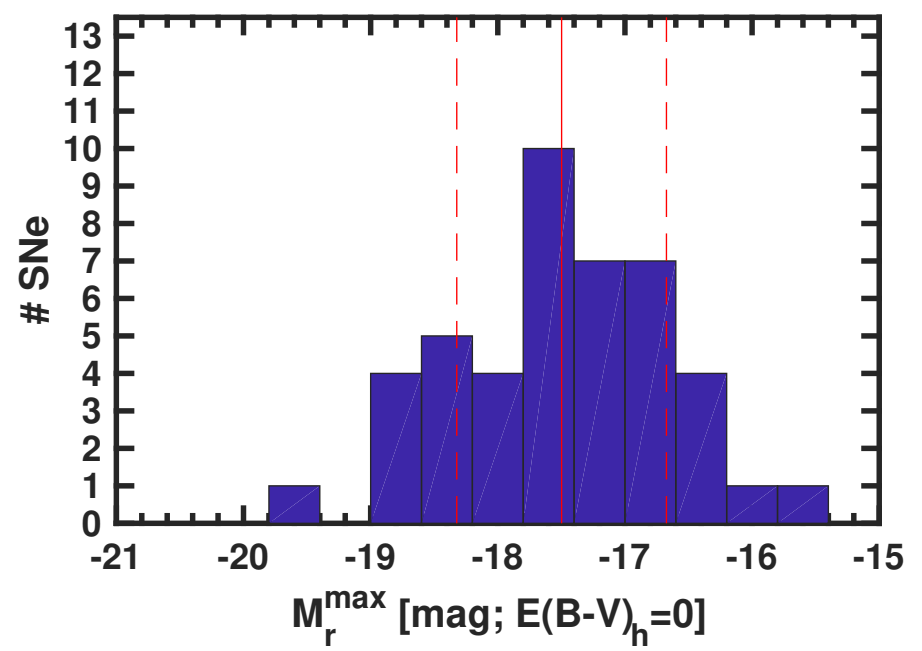

Table 3. Absolute magnitudes for $40 \mathrm{SNe}$ in the $r$ band (corrected for distance modulus and MW extinction), compared with the absolute magnitudes in the $r$ band obtained when including the contribution from the host extinction with both methods, from the $\mathrm{Na} I \mathrm{D}$ absorption and the $g-r$ method.

\begin{tabular}{|c|c|c|c|}
\hline SN & $\begin{array}{c}M_{r}^{\text {peak }} \\
\text { no } \mathrm{EBV}_{\text {host }} \\
(\mathrm{mag})\end{array}$ & $\begin{array}{c}M_{r}^{\text {peak }} \\
\mathrm{EBV}_{\text {host }} \text { from Na I D } \\
(\mathrm{mag})\end{array}$ & $\begin{array}{c}M_{r}^{\text {peak }} \\
\mathrm{EBV}_{\text {host }} \text { from } g-r \\
(\mathrm{mag})\end{array}$ \\
\hline 09dh & -18.88 & -19.30 & -19.25 \\
\hline 09ut & -16.53 & -16.53 & -17.16 \\
\hline 10bip & -17.52 & -17.67 & -17.52 \\
\hline 10hfe & -16.75 & -16.75 & -16.75 \\
\hline 10hie & -17.84 & -17.89 & -18.68 \\
\hline 10lbo & -17.56 & -17.56 & -17.56 \\
\hline $10 \mathrm{osn}$ & -16.90 & -16.90 & -17.05 \\
\hline 10tqi & -17.15 & -17.57 & -17.62 \\
\hline 10yow & -16.78 & -17.23 & -17.33 \\
\hline $10 \mathrm{zcn}$ & -16.55 & -16.85 & -18.19 \\
\hline 11bli & -17.42 & -17.67 & -18.69 \\
\hline $11 \mathrm{bov}$ & -18.32 & -18.44 & -18.32 \\
\hline $11 \mathrm{jgj}$ & -17.32 & -17.90 & -18.00 \\
\hline $11 \mathrm{klg}$ & -17.26 & -17.26 & -17.99 \\
\hline $11 \mathrm{mnb}$ & -18.29 & -18.49 & -18.50 \\
\hline $11 \mathrm{mwk}$ & -18.39 & -18.44 & -18.39 \\
\hline 11rka & -18.64 & -18.85 & -19.40 \\
\hline 12 cjy & -16.93 & -16.93 & -16.93 \\
\hline $12 \mathrm{dcp}$ & -17.09 & -17.09 & -18.07 \\
\hline $12 \mathrm{dtf}$ & -17.67 & -17.78 & -18.56 \\
\hline $12 \mathrm{fgw}$ & -17.77 & -17.77 & -17.77 \\
\hline 12 gty & -19.73 & -19.81 & -19.84 \\
\hline $12 \mathrm{gzk}$ & -18.65 & -19.01 & -19.19 \\
\hline $12 \mathrm{hvv}$ & -17.37 & -17.57 & -19.18 \\
\hline $12 \mathrm{jxd}$ & -16.04 & -16.87 & -17.14 \\
\hline $12 \mathrm{ktu}$ & --16.38 & -16.59 & -17.25 \\
\hline $13 \mathrm{ab}$ & -16.91 & -17.02 & -16.91 \\
\hline 13aot & -16.90 & -17.00 & - \\
\hline $13 \mathrm{cuv}$ & -17.26 & -17.30 & -17.94 \\
\hline $13 \mathrm{dht}$ & -16.87 & -17.03 & -17.38 \\
\hline $13 \mathrm{djf}$ & -15.45 & -15.53 & -16.92 \\
\hline 14bpy & -17.78 & -17.78 & -17.78 \\
\hline 14 fuz & -17.40 & -17.52 & -17.41 \\
\hline $14 \mathrm{gqr}$ & -17.41 & -17.41 & -17.41 \\
\hline $14 \mathrm{jhf}$ & -17.82 & -18.62 & - \\
\hline $14 y m$ & -17.37 & -17.76 & -17.94 \\
\hline $15 \mathrm{acp}$ & -18.77 & -19.10 & -19.62 \\
\hline $15 \mathrm{dtg}$ & -18.00 & -18.00 & -18.28 \\
\hline $16 \mathrm{flq}$ & -17.90 & -18.51 & -18.49 \\
\hline 16hgp & -18.48 & -18.48 & -18.65 \\
\hline
\end{tabular}

Fig. 12. Histogram representation of the absolute magnitudes at peak in the $r$-band distribution of the sample. Upper panel: distribution obtained correcting for the distance and the MW extinction. Middle panel: distribution obtained including also the extinction from the host galaxy, estimated through the NaID absorption. Bottom panel: distribution obtained including instead the host extinction from the colors.

$-18.07 \pm 0.84 \mathrm{mag}$. All values for each $\mathrm{SN}$ in the sample are reported in Table 3. We notice how PTF12gty is the brightest SN in the sample with an absolute peak magnitude of -19.81 .

The absolute magnitude ranges available in the literature are $M_{r}^{\text {peak }}=-18.26 \pm 0.21 \mathrm{mag}$ (Taddia et al. 2015); $M_{r}^{\text {peak }}=$

$-17.64 \pm 0.26 \mathrm{mag}$ (Taddia et al. 2018b) and $M_{r}^{\text {peak }}=-18.3 \pm$ $0.6 \mathrm{mag}$ (Drout et al. 2011). The average peak magnitude in the $r$ band estimated for our sample is thus in agreement with the ones from the literature. We compared these values also with the (i)PTF sample of SNe Ic-BL (Taddia et al. 2019b) where the average peak magnitude is $-18.7 \pm 0.7 \mathrm{mag}$. Our SNe Ic are on average fainter than the SNe Ic-BL. We investigated the absolute $r$-band magnitude peak vs. $\Delta m_{15}(r)$ behavior, to test if there is a Phillips-like relation as for SNe Ia (Phillips 1993). We found that SNe Ic do not show any clear correlation (see Fig. 13).

This is in agreement with previous studies on SE SNe (Prentice et al. 2016; Lyman et al. 2016; Drout et al. 2011). Also 


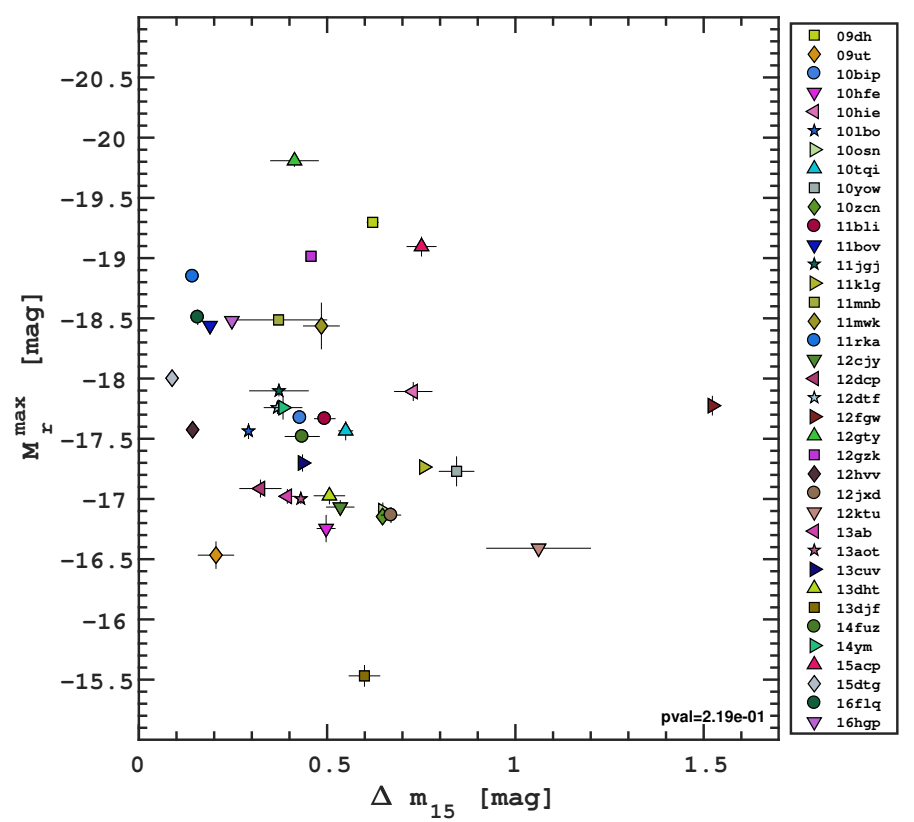

Fig. 13. Peak absolute $r$-band magnitude vs. $\Delta m_{15}$. The plot shows no correlation.

a dedicated study on SNe Ic-BL has shown that there is no evidence for such a correlation (Taddia et al. 2019b).

For SNe having data more than 70 days past peak, we also measured the slope at late epochs. We investigated the $\Delta m_{15}(r)$ vs. the slope and we did not see any clear correlation. This is not in agreement with what Taddia et al. (2018b) found in their work. Our $g$-band peak magnitudes for $19 \mathrm{SNe}$ span the interval -15.86 to -18.91 mag when the host extinction is not taken into account and it ranges from -17.10 to $-19.51 \mathrm{mag}$ when included, with an average value of $-17.99 \pm 0.69 \mathrm{mag}$. Finally, if we consider the extinction we get from the $g-r$ method the interval is -17.04 to $-19.44 \mathrm{mag}$. In this case the average is $-18.39 \pm 0.65 \mathrm{mag}$. The average value for the peak magnitude in the $g$-band is in agreement with the $-17.28 \pm 0.24$ found by Taddia et al. (2018b).

\subsection{Explosion epochs and rise times}

The separation between last non-detection and first detection for all the SNe of the sample varies in the interval 1-30 days, with the exception of six $\mathrm{SNe}^{8}$ that do not have last non-detection within 50 days prior the first detection. In order to estimate the explosion epochs for each $\mathrm{SN}$, we compare their $r$-band light curves with the $r$-band light curve of iPTF13djf. This supernova has a good photometric coverage and well determined explosion epoch, with a limit on the discovery date of only \pm 1 day. Since the explosion epoch of iPTF13djf is well constrained, as is the peak epoch in the $r$ band, we use it as a template and the stretch of the best fit allows us to infer the explosion epoch for all other SNe in the sample. The light curve of iPTF13djf is stretched in time and shifted in magnitude to fit our SN light curves until +30 days post peak. The estimated explosion epochs were checked against the pre-explosion limits for consistency. We adopted \pm 2 days as a conservative estimate of the uncertainties on the explosion epochs.

8 PTF10hie, PTF11klg, iPTF12cjy, iPTF14jhf, iPTF14ym and iPTF16flq.
In a few cases when this method did not give results consistent with the pre-explosion limits, we assume the last nondetection as the explosion epoch ${ }^{9}$. We note that in these cases the values we will estimate for the rise time will have to be considered as an upper limit. When an estimate for the SN explosion epoch was available from literature, we adopted the latter as our explosion epoch. This was the case for PTF11mnb (Taddia et al. 2018a), iPTF14gqr (De et al. 2018) and iPTF15dtg (Taddia et al. 2016). The best fits and the obtained explosion epochs are shown in Fig. 14.

The inferred explosion epochs are reported in Table 4. The explosion epochs and the epochs of the maximum in $r$ band allow us to compute the rest-frame $r$-band rise time, these are provided in Table 4. The average rise time we get is 25.3 days which is somewhat higher than the 16.8 days found by Lyman et al. (2016) and the 13.3 days found by Taddia et al. (2018b). This is most likely due to the fact we have more slow rising $\mathrm{SNe}$ than in their samples.

\section{Construction of the bolometric light curves}

Modeling of the bolometric light curves can help derive parameters on the supernova progenitors and on the explosion physics. To accomplish this, we need to estimate the explosion epochs and construct the bolometric light curves.

\subsection{Bolometric lightcurves}

Due to the lack of a complete multiband coverage, in particular at early epochs, we used the absolute $r$-band light curves and the fit of the $g-r$ color evolution to compute the bolometric light curves, making use of the bolometric corrections for SE SNe presented by Lyman et al. (2014). In this way we are able to create bolometric light curves covering all the phases. The bolometric light curves of $12 \mathrm{SNe}^{10}$ were built by applying the bolometric correction directly to the $g$ band, which is what is needed for the method of Lyman et al. (2014). For the other $30 \mathrm{SNe}$, we interpolate the $g$ band from the $r$ band and then applied the bolometric correction. Only for iPTF13aot and iPTF14jhf were we unable to build a bolometric light curve due to a lack of $g-r$ evolution and these are therefore excluded from the analysis. The final bolometric light curves as a function of days since explosion are shown in Fig. 15.

The systematic uncertainties due to the bolometric correction $(0.076 \mathrm{mag})$ and on the distance $(0.15 \mathrm{mag})$ are not included in the errors of each bolometric light curve.

\subsection{Analysis of the bolometric light curve shape}

We fit the bolometric light curves with the Contardo function also used in Sect. 4.2. The best fits are shown in the plot as solid lines in Fig. 15. Following the same analysis as for the $r$ band, this allows us to measure some properties of the shape of the bolometric light curves, such as the peak magnitude, the peak epoch, $\Delta m_{-10}, \Delta m_{15}$ as well as the linear decline slope. We present all these parameters in Table 2. Our sample peak magnitudes span the interval -16.10 to $-19.78 \mathrm{mag}$ giving an average of $\left\langle M_{\mathrm{bol}}^{\mathrm{peak}}\right\rangle=-17.62 \pm 0.94 \mathrm{mag}$. These values are in

\footnotetext{
9 We assumed the last non detection as explosion epoch for PTF09dh, PTF10hfe, PTF10tqi, PTF10zcn and PTF12gzk.

10 PTF11bli, PTF11bov, PTF11hyg, PTF11lmn, PTF11mnb, iPTF 14fuz, iPTF14gao, iPTF14gqr, iPTF15acp, iPTF15cpq, iPTF15dtg and iPTF16hgp.
} 


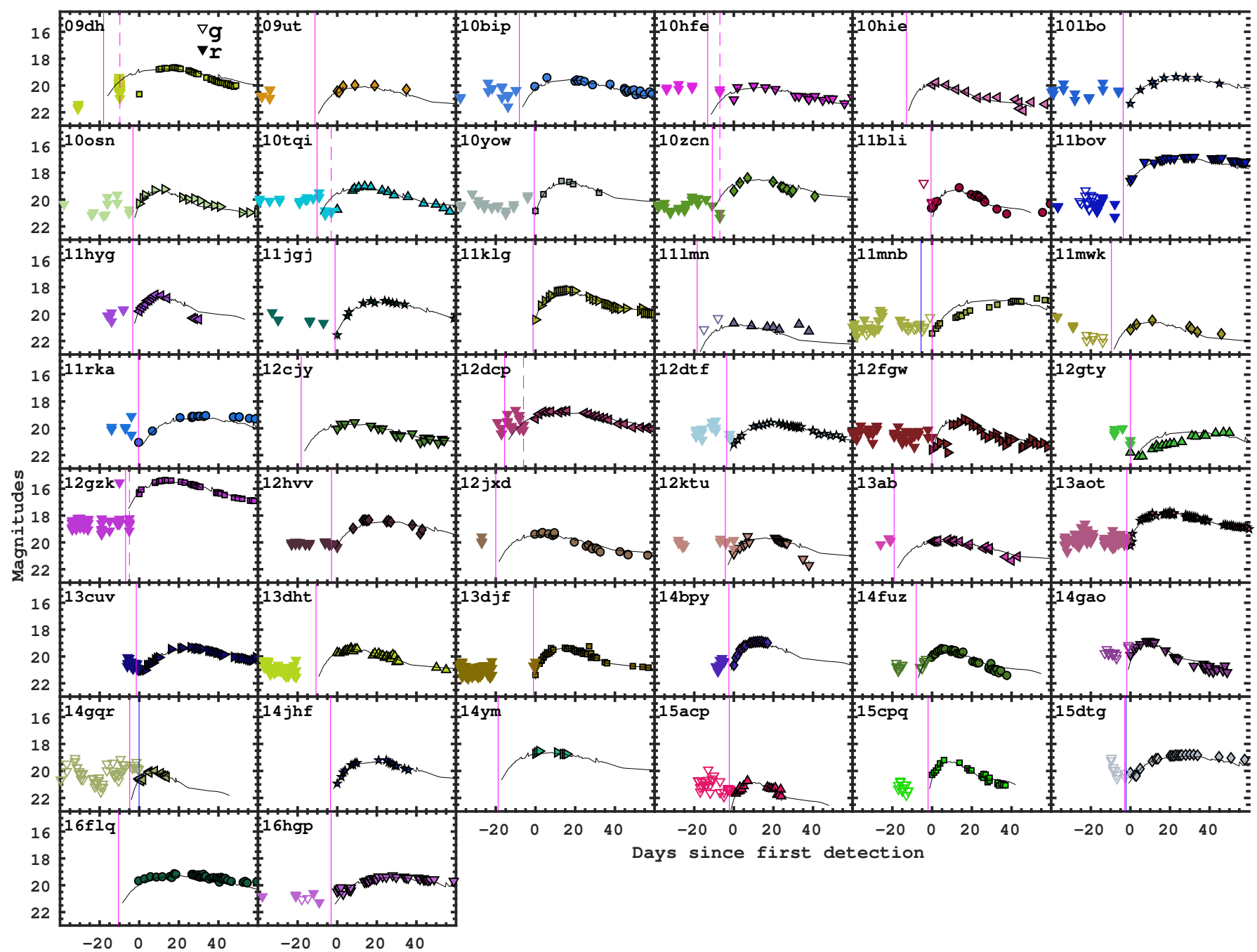

Fig. 14. Fit of the light curve of iPTF13djf to the other SNe of the sample to estimate the explosion epoch. The open symbols represents the preexplosion limits. Solid magenta lines represent the explosion epochs estimated from the fit. Dashed magenta lines represent the last non-detection assumed as explosion epoch. Blue solid lines represent explosion epochs available from literature.

agreement with the ones available in literature (Drout et al. 2011; Prentice et al. 2016; Lyman et al. 2016; Taddia et al. 2018b) We investigated the same correlations as for the $r$ band, they are presented in Fig. 16. We find the same correlations as for the $r$-band. We notice that the similarity of relations found among rise and decline parameters in $r$-band and bolometric light curve is likely due to the fact that the flux in $r$ gives a close representation of the bolometric flux at most epochs. We also estimated the rise times for the bolometric light curves in the same way as we did for the $r$ band and these values are reported in Table 4 .

\section{Supernova spectra}

This work is focused on the photometric analysis of a sample of SNe Ic, a study of the spectroscopic properties was presented in Fremling et al. (2018). However, one of the aims is to use these light curves to estimate explosion parameters (Sect. 7), and in order to break the degeneracy between explosion energy and ejecta mass such an analysis requires an estimate of the photospheric velocity. This will be presented here. We have a total of 177 spectra for the overall sample. All the spectra will become available via the WiseRep archive (Yaron \& Gal-Yam 2012).
They were already discussed and analyzed in Fremling et al. (2018) and will be provided in a larger data-release by Fremling et al. (in prep.).

In order to determine the photospheric velocities for the $\mathrm{SNe}$ in our sample we estimate the expansion velocities using the Fe II $\lambda 5169$ line. These velocities were evaluated from the minima of the P Cygni profile of the Fe II $\lambda 5169$ for all the available spectra. For six $\mathrm{SNe}^{11}$ it was not possible to estimate the Fe II $\lambda 5169$ velocities as the $\mathrm{S} / \mathrm{N}$ of the spectra were too low. Since we aim to build up a functional form for the general trend for normal SNe Ic, we excluded PTF12gzk as it is known from the literature to be a high velocity SN (Horesh et al. 2013). We note that this exclusion is only for the purpose to limit the velocity dispersion while determining the functional form, the SN velocities will be estimated and included in the final analysis. The time evolution of the Fe II $\lambda 5169$ velocities for the $37 \mathrm{SNe}$ so selected is presented in Fig. 17, where the magenta solid line shows the power law which best represents the trend shown by the overall velocities.

This power-law trend for Fe II $\lambda 5169$ is in agreement with the trend found by Taddia et al. (2018b) for SE SNe, the functional form we found is $v(t) \propto\left(t-t_{0}\right)^{-0.30}$, where $t_{0}$ represents the

${ }_{11}$ PTF09dh, PTF09ut, PTF11jgj, PTF12cjy, PTF12fgw and iPTF13djf. 
explosion epoch. In Fig. 17 we also compare the Fe II $\lambda 5169$ evolution with the trend found by Modjaz et al. (2016). Their velocities are lower than our best fits at early epochs. A polynomial fit is also presented.

The photospheric velocities required to estimate the explosion parameters are the Fe II $\lambda 5169$ velocities at peak. This is not available for every $\mathrm{SN}$ in the sample. We then use the general trend found for the overall sample and assume that it represents the velocity evolution for each individual SN. We thus apply our power-law as a template to every $\mathrm{SN}$, shifting it to the available velocity values for the individual SNe. Once it has been shifted, we can extrapolate the value of the Fe II $\lambda 5169$ velocity at peak. We estimate the average velocity at peak of the $\mathrm{SNe}$ of our sample and adopted this average value to be the velocity for the six SNe for which we were unable to measure the Fe II $\lambda 5169$ velocity. The power-law method is also applied to the velocities estimated for PTF12gzk, as these velocities show a similar trend but at higher values. The uncertainties on the peak velocity were assumed to be $10 \%$ of the estimated value. In this way we get a full set of velocities at peak for the $42 \mathrm{SNe}$ of the sample that will be used in Sect. 7 to estimate the explosion parameters. The estimated velocities at peak are presented in Table 5. We notice how PTF10bip presents higher velocities compared to the average of the sample, this could explain the Ic/Ic-BL classification from Modjaz et al. (2020).

\section{Explosion parameters}

In order to estimate the explosion parameters, we fit the bolometric light curves with an Arnett model (Arnett 1982). The method we followed to perform the fit is presented in Taddia et al. (2018b). We performed the fits on the early epochs of the light curves, $\lesssim 60$ days after peak, during the photospheric phase of the SNe. The parameters we can estimate from this modeling are the ${ }^{56} \mathrm{Ni}$ mass $\left(M_{56} \mathrm{Ni}\right)$, the kinetic energy of the explosion $\left(E_{\mathrm{K}}\right)$ and the ejecta mass $\left(M_{\mathrm{ej}}\right)$. We assume that the $\mathrm{SN}$ ejecta have spherical symmetry and uniform density; we also use $E / M=(3 / 10) V^{2}$, where $V$ is the appropriate ejecta velocity at peak as discussed in Sect. 6 (see Valenti et al. 2008). We furthermore assumed a constant opacity $\kappa=0.07 \mathrm{~cm}^{2} \mathrm{~g}^{-1}$, as is often done in the literature for SE SN samples. The Arnett fit for each $\mathrm{SN}$ is shown in Fig. 18.

The estimated values for $M_{56 \mathrm{Ni}}, M_{\mathrm{ej}}$, and $E_{\mathrm{K}}$ are listed in Table 6 . The uncertainties on $M_{56}{ }^{5 i}$ are mostly due to the uncertainty in the SN distances. The uncertainties in $M_{\mathrm{ej}}$ and $E_{\mathrm{K}}$ instead depend mostly on the uncertainty on the expansion velocity. We notice that the Arnett fit gives a particular high value of $M_{56} \mathrm{Ni}$ for PTF12gty $\left(\sim 3 M_{\odot}\right)$. We note that PTF12gty has been an outlier for most of the analysis in this work, in particular it has the largest redshift, the highest peak absolute magnitude and longest rise time. It also has the lowest velocity at peak. We conclude that this SN is most likely a SLSN, as discussed in De Cia et al. (2018) and Quimby et al. (2018).

It will therefore be excluded from the estimates of the average explosion parameters. This reduces the final sample of $\mathrm{SNe}$ Ic to 41 . We then obtained average values of $\left\langle M_{\mathrm{ej}}\right\rangle=4.50 \pm$ $0.79 M_{\odot},\left\langle E_{\mathrm{K}}\right\rangle=1.79 \pm 0.29$ foe $\left(1\right.$ foe $\left.=10^{51} \mathrm{erg}\right)$, and $\left\langle M_{56} \mathrm{Ni}\right\rangle=$ $0.19 \pm 0.03 M_{\odot}$ where the errors are the weighted errors.

In Fig. 19 we plot each estimated parameter against the others. We identify a correlation between $M_{\mathrm{ej}}$ and $E_{\mathrm{K}}$ (see bottom panel). We also notice a correlation between the $M_{\mathrm{ej}}$ and $M_{56 \mathrm{Ni}}$, and between $M_{56 \mathrm{Ni}}$ and $E_{\mathrm{K}}$. We note that the small variation of
Table 4. Estimated explosions time ( $\left.t_{\text {explo }}\right)$, the first detection $\left(t_{\text {firstdet }}\right)$, estimated rise times in the $r$ band $\left(t_{\text {rise }}(r)\right)$ compared with the ones from the bolometric light curves $\left(t_{\text {rise }}(\right.$ bolo) $)$ for the $44 \mathrm{SNe}$ of our sample.

\begin{tabular}{|c|c|c|c|c|}
\hline SN & $\begin{array}{l}t_{\text {explo }} \\
\text { (JD) }\end{array}$ & $\begin{array}{c}t_{\text {firstdet }} \\
\text { (JD) }\end{array}$ & $\begin{array}{l}t_{\text {rise }}(r) \\
\text { (days) }\end{array}$ & $\begin{array}{c}t_{\text {rise }}(\text { bolo }) \\
\text { (days) }\end{array}$ \\
\hline 09dh & 2454929.02 & 2454938.80 & 23.75 & 20.70 \\
\hline 09ut & 2454994.62 & 2455005.82 & 18.88 & 35.54 \\
\hline 10bip & 2455213.75 & 2455221.85 & 17.09 & 13.90 \\
\hline 10hfe & 2455324.83 & 2455331.87 & 15.57 & 12.44 \\
\hline 10hie & 2455323.06 & 2455335.96 & 12.38 & 15.80 \\
\hline 10lbo & 2455349.10 & 2455352.81 & 26.59 & 21.73 \\
\hline 10 osn & 2455385.78 & 2455388.98 & 11.95 & 10.89 \\
\hline 10tqi & 2455426.96 & 2455429.98 & 16.00 & 13.66 \\
\hline 10yow & 2455467.01 & 2455467.61 & 13.41 & 12.36 \\
\hline $10 \mathrm{zcn}$ & 2455471.76 & 2455478.82 & 15.54 & 16.87 \\
\hline $11 \mathrm{bli}$ & 2455629.49 & 2455635.75 & 15.97 & 17.13 \\
\hline $11 \mathrm{bov}$ & 2455648.11 & 2455651.71 & 36.34 & 25.13 \\
\hline 11 hyg & 2455735.62 & 2455738.92 & 14.82 & 14.96 \\
\hline $11 \mathrm{jgj}$ & 2455764.66 & 2455765.76 & 20.85 & 17.90 \\
\hline $11 \mathrm{klg}$ & 2455791.67 & 2455792.87 & 16.94 & 15.47 \\
\hline $111 \mathrm{mn}$ & 2455794.29 & 2455801.74 & 16.97 & 21.19 \\
\hline $11 \mathrm{mnb}$ & 2455804.34 & 2455809.88 & 53.77 & 54.87 \\
\hline $11 \mathrm{mwk}$ & 2455811.23 & 2455820.83 & 16.55 & 14.31 \\
\hline 11rka & 2455895.61 & 2455896.01 & 32.54 & 33.67 \\
\hline $12 \mathrm{cjy}$ & 2455999.74 & 2456017.94 & 25.28 & 21.74 \\
\hline $12 \mathrm{dcp}$ & 2456027.93 & 2456033.96 & 19.62 & 28.69 \\
\hline $12 \mathrm{dtf}$ & 2456033.35 & 2456036.95 & 21.72 & 24.95 \\
\hline $12 \mathrm{fgw}$ & 2456077.64 & 2456077.74 & 14.90 & 13.62 \\
\hline 12 gty & 2456086.82 & 2456086.82 & 72.38 & 52.64 \\
\hline $12 \mathrm{gzk}$ & 2456132.86 & 2456137.82 & 19.31 & 16.55 \\
\hline $12 \mathrm{hvv}$ & 2456148.97 & 2456151.87 & 16.51 & 28.63 \\
\hline $12 \mathrm{jxd}$ & 2456206.04 & 2456226.04 & 23.31 & 21.50 \\
\hline $12 \mathrm{ktu}$ & 2456227.59 & 2456231.89 & 17.24 & 19.50 \\
\hline $13 \mathrm{ab}$ & 2456308.78 & 2456327.88 & 23.89 & 19.34 \\
\hline 13aot & 2456394.05 & 2456395.85 & 22.09 & - \\
\hline $13 \mathrm{cuv}$ & 2456510.40 & 2456511.90 & 25.75 & 26.98 \\
\hline $13 \mathrm{dht}$ & 2456531.98 & 2456542.68 & 18.64 & 16.41 \\
\hline $13 \mathrm{djf}$ & 2456542.76 & 2456543.76 & 14.80 & 12.09 \\
\hline 14 bpy & 2456821.20 & 2456823.70 & 13.78 & 11.19 \\
\hline 14 fuz & 2456912.89 & 2456925.88 & 13.88 & 16.83 \\
\hline $14 \mathrm{gao}$ & 2456911.17 & 2456912.97 & 14.85 & 13.73 \\
\hline $14 \mathrm{gqr}$ & 2456944.25 & 2456944.34 & 7.18 & 6.06 \\
\hline 14jhf & 2457008.50 & 2457011.90 & 25.08 & - \\
\hline $14 \mathrm{ym}$ & 2456692.23 & 2456711.03 & 22.48 & 18.23 \\
\hline $15 \mathrm{acp}$ & 2457105.67 & 2457107.97 & 14.56 & 12.74 \\
\hline $15 \mathrm{cpq}$ & 2457268.87 & 2457270.87 & 15.69 & 12.95 \\
\hline $15 \mathrm{dtg}$ & 2457333.45 & 2457333.93 & 37.41 & 19.88 \\
\hline $16 f l q$ & 2457617.46 & 2457627.96 & 25.57 & 19.34 \\
\hline 16hgp & 2457678.69 & 2457681.79 & 30.90 & 21.76 \\
\hline
\end{tabular}

the velocity's range is possibly driving the correlation between the energy and the ejecta mass. The probability density function (PDF) of the three explosion parameters are shown in Fig. 20.

It shows, for all parameters, that most of the $\mathrm{SNe}$ are distributed around a common peak, but there are also evidence for distributions towards higher values in all three parameters. In Table 1 we present $8 \mathrm{SNe}$ with a type Ibc classification ${ }^{12}$. We could not build the bolometric light curve for iPTF14jhf, which 12 PTF09ut, PTF11bli, PTF11lmn, PTF11mwk, iPTF14fuz, iPTF14jhf,
iPTF15cpq and iPTF16flq. 


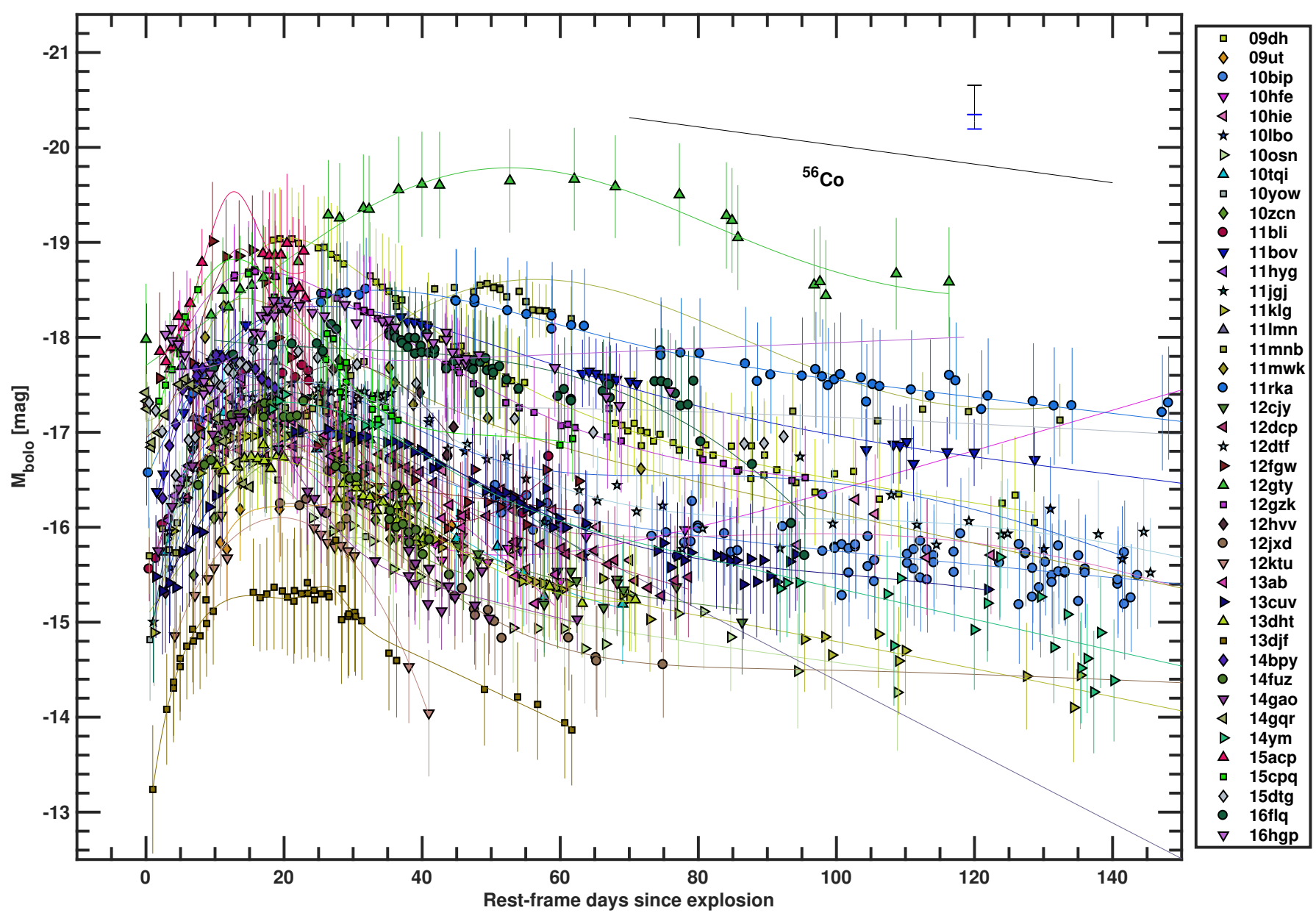

Fig. 15. Bolometric light curves of 42 Type Ic SNe. The solid lines represent the Contardo fits performed on every individual light curve. The slope of the radioactive cobalt decay, 0.098 mag per day is illustrated in the upper right corner. There we also include a representative error bar that includes the uncertainty in distance, and extinction, respectively, which are not included in the errors on the data points.

leaves us with $7 \mathrm{SNe}$ Ibc. If we exclude these from our sample, we obtained average values of $\left\langle M_{\mathrm{ej}}\right\rangle=4.65 \pm 0.93 M_{\odot}$, $\left\langle E_{\mathrm{K}}\right\rangle=1.88 \pm 0.34$ foe, and $\left\langle M_{56} \mathrm{Ni}\right\rangle=0.19 \pm 0.03 M_{\odot}$ where the errors are the weighted errors. In Sect. 2, we mentioned that $6 \mathrm{SNe}$ show broader light curves compared to the rest of the sample, which will be discussed separately (Karamehmetoglu et al., in prep.). Among these 6 SNe we already excluded PTF12gty as it is most likely a SLSN, leaving us with $5 \mathrm{SNe}$ Ic showing a broad light curve. If we exclude these $\mathrm{SNe}^{13}$ we obtain averages $\left\langle M_{\mathrm{ej}}\right\rangle=3.57 \pm 0.40 M_{\odot},\left\langle E_{\mathrm{K}}\right\rangle=1.74 \pm 0.33$ foe, and $\left\langle M_{56 \mathrm{Ni}}\right\rangle=0.16 \pm 0.02 M_{\odot}$ where again the errors represent the weighted errors. Excluding the $5 \mathrm{SNe}$ with broad light curves clearly gives lower average values for $M_{\mathrm{ej}}$ and $M_{56} \mathrm{Ni}$. If we furthermore exclude from the average the peculiar fast ultrastripped iPTF14gqr (De et al. 2018) we get $\left\langle M_{\mathrm{ej}}\right\rangle=3.67 \pm 0.39 M_{\odot}$, $\left\langle E_{\mathrm{K}}\right\rangle=1.78 \pm 0.32$ foe and $\left\langle M_{56 \mathrm{Ni}}\right\rangle=0.16 \pm 0.02 M_{\odot}$. These average values, now based on 34 normal SNe Ic, are still consistent with the previous ones within the uncertainties. We estimated the explosion parameters also in the case when the bolometric light curves were built considering the host extinction estimated from the $g-r$ color evolution. In this case we got $\left\langle M_{\mathrm{ej}}\right\rangle=3.17 \pm 0.99,\left\langle E_{\mathrm{K}}\right\rangle=1.12 \pm 0.23$, and $\left\langle M_{56} \mathrm{Ni}_{\mathrm{i}}\right\rangle=0.77 \pm 0.25$ where again the errors are weighted sigma. The average $\left\langle M_{\mathrm{ej}}\right\rangle$, as well as the $\left\langle E_{\mathrm{K}}\right\rangle$, are lower but still consistent with the previous

\footnotetext{
${ }^{13}$ PTF11mnb, PTF11rka, iPTF15dtg, iPTF16flq and iPTF16hgp.
}

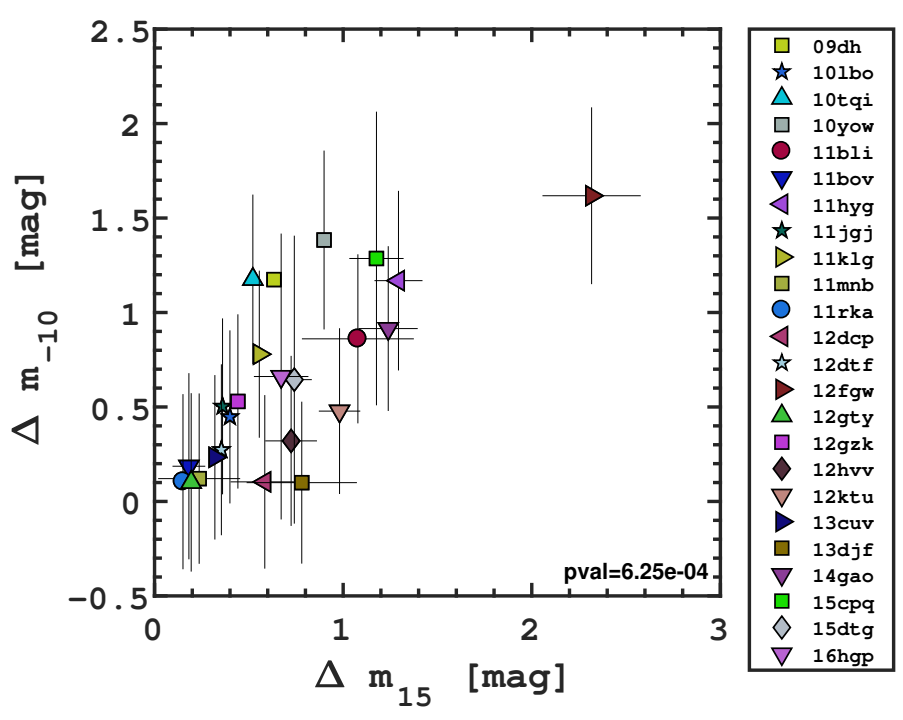

Fig. 16. Bolometric light curve shape: $\Delta m_{15}$ vs. $\Delta m_{-10}$. The plot does show a correlation, as also found in the $r$-band.

measurements within the uncertainties but $\left\langle M_{56} \mathrm{Ni}\right\rangle$ is higher. This is not surprising since we got systematically higher values of the extinction with this method and the $M_{56} \mathrm{Ni}$ depends mainly from the peak luminosity. 


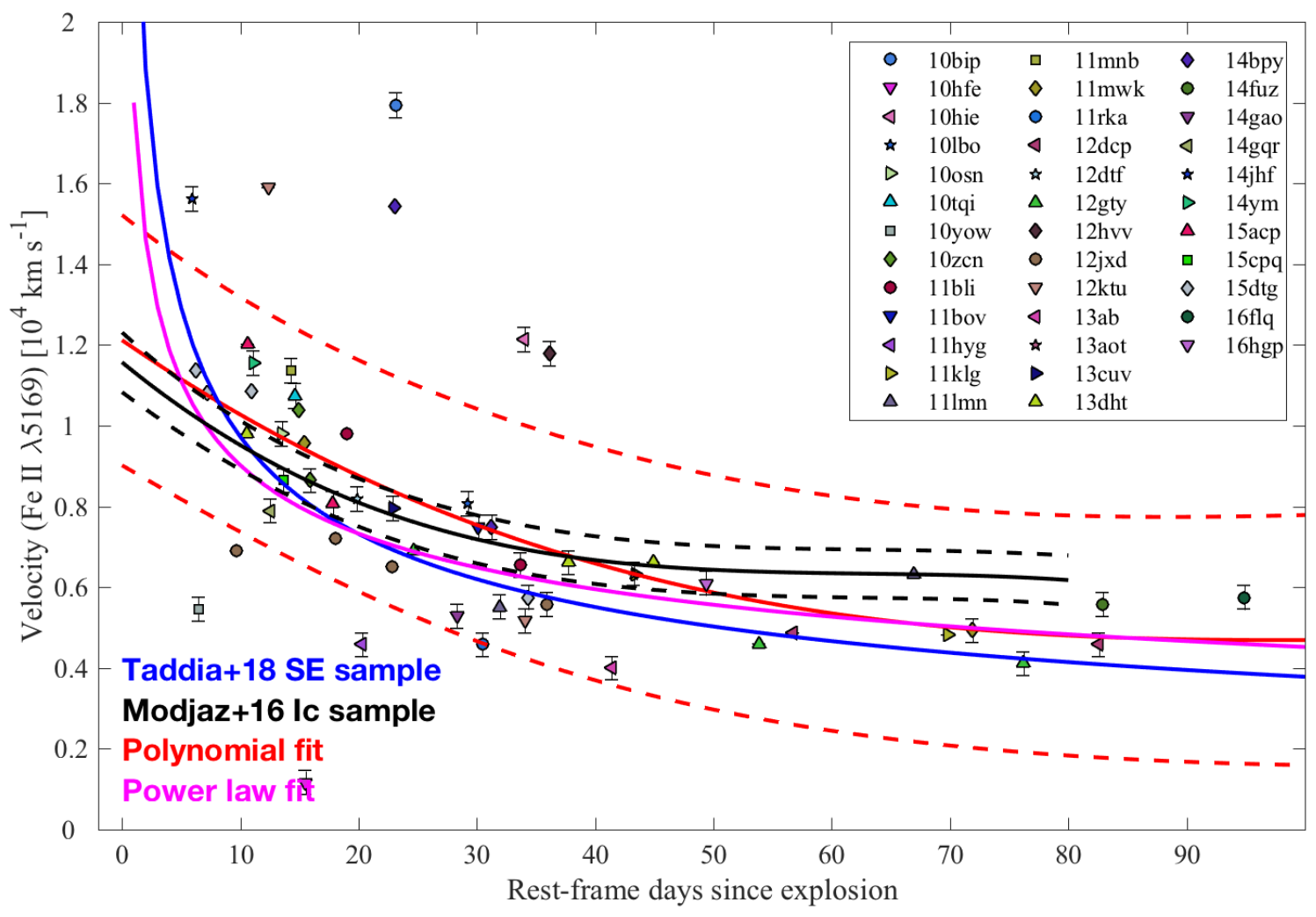

Fig. 17. Fe II $\lambda 5169$ velocity evolution for $37 \mathrm{SNe}$ Ic from the sample (see Sect. 6 for the selection criteria). The magenta solid line represents the power law that fits the evolution with time. The blue solid line represents the trend found by Taddia et al. (2018b) and is similar to the one found in this work. The black lines represent the polynomial fit found by Modjaz et al. (2016). As a comparison, we fitted the data also with a polynomial fit, here shown in red.

\section{Discussion and conclusions}

PTF and iPTF allowed for a larger, untargeted, and more homogeneous data set as compared with other SN Ic samples, and for this sample we also have good constraints on the explosion epochs.

We investigated two different methods to estimate the host extinction. First we inspected the spectra to look for NaID absorption and using Taubenberger et al. (2006) we calculated the $E(B-V)$. This method is dependent on the $\mathrm{S} / \mathrm{N}$ and resolution of the spectrum. The second method is based on the $g-r$ colour evolution and is described in Stritzinger et al. (2018). This method assumes that SE SNe show an intrinsically homogeneous color evolution in the range 0-20 days past peak. We compare the results of these methods in Fig. 10, which shows that the extinction estimated through the color evolution is generally higher.

We adopted the extinction estimated from the NaID for the overall analysis, but also compared how the peak magnitudes would change if we had adapted the other method. The average absolute peak magnitude is $\left\langle M_{r}^{\text {peak }}\right\rangle=-17.71 \pm 0.85$ mag. In case we adopt the extinction from the $g-r$ evolution we get $\left\langle M_{r}^{\text {peak }}\right\rangle=-18.03 \pm 0.79 \mathrm{mag}$. The effect on the overall peak magnitude distribution are shown in Fig. 12, where accounting for higher extinction as suggested by the Stritzinger et al. (2018) method shifts the overall sample towards brighter magnitudes.

We investigated the light curve shape in both the $r$ band and for the bolometric light curves. We looked for correlations among the main parameters: magnitude at peak, $\Delta m_{-10}, \Delta m_{15}$, $\Delta m_{40}$ and slope. In both cases, we found a correlation between $\Delta m_{15}$ and $\Delta m_{-10}$, implying that slow-rising $\mathrm{SNe}$ are also slow decliners. We see a correlation also among $\Delta m_{40}$ vs. $\Delta m_{15}$ and among $\Delta m_{40}$ vs. $\Delta m_{-10}$.

We fitted the bolometric light curves with an Arnett model (Arnett 1982) to estimate the explosion parameters. We obtained average values of $\left\langle M_{\mathrm{ej}}\right\rangle=4.39 \pm 0.31 M_{\odot},\left\langle E_{\mathrm{K}}\right\rangle=1.71 \pm 0.16$ foe, and $\left\langle M_{56 \mathrm{Ni}}\right\rangle=0.19 \pm 0.05 M_{\odot}$, when including all the $41 \mathrm{SNe}$ Ic for which we could estimate these parameters. We searched for correlations among the explosion parameters and identify a correlation between $M_{\mathrm{ej}}$ and $E_{\mathrm{K}}$. We also notice a correlation between the $M_{\mathrm{ej}}$ and $M_{56 \mathrm{Ni}}$, and between $M_{56 \mathrm{Ni}}$ and $E_{\mathrm{K}}$.

\subsection{Comparison with the literature}

Some of the $\mathrm{SNe}$ in this sample have already been discussed in the literature, and we will here compare our results with those available in these publications. SNe PTF09dh, PTF11bli, PTF11jgj, PTF11klg, PTF11rka and PTF12gzk were presented in Prentice et al. (2016). Our estimated $M_{56} \mathrm{Ni}$ values for these $\mathrm{SNe}$ are in agreement with the ones provided in their work. PTF12gzk is discussed in Ben-Ami et al. (2012), in which they noted that this $\mathrm{SN}$ showed some aspects in-between $\mathrm{SNe}$ Ic and SNe Ic-BL. They conclude that the mass of the progenitor star is $25-35 M_{\odot}$. We get quite high values for the ejecta mass which might point towards a massive progenitor star as found by Ben-Ami et al. (2012). PTF11bov is also known as SN 2011bm and was presented in Valenti et al. (2012) where they infer an initial mass for the progenitor of $30-50 M_{\odot}$. The ejecta mass we derive is close to the lower end of the interval they present in their work. iPTF15dtg was first introduced in Taddia et al. (2016) and investigated further in Taddia et al. (2019a). In their first work they concluded that the peculiar long rise of this SN 


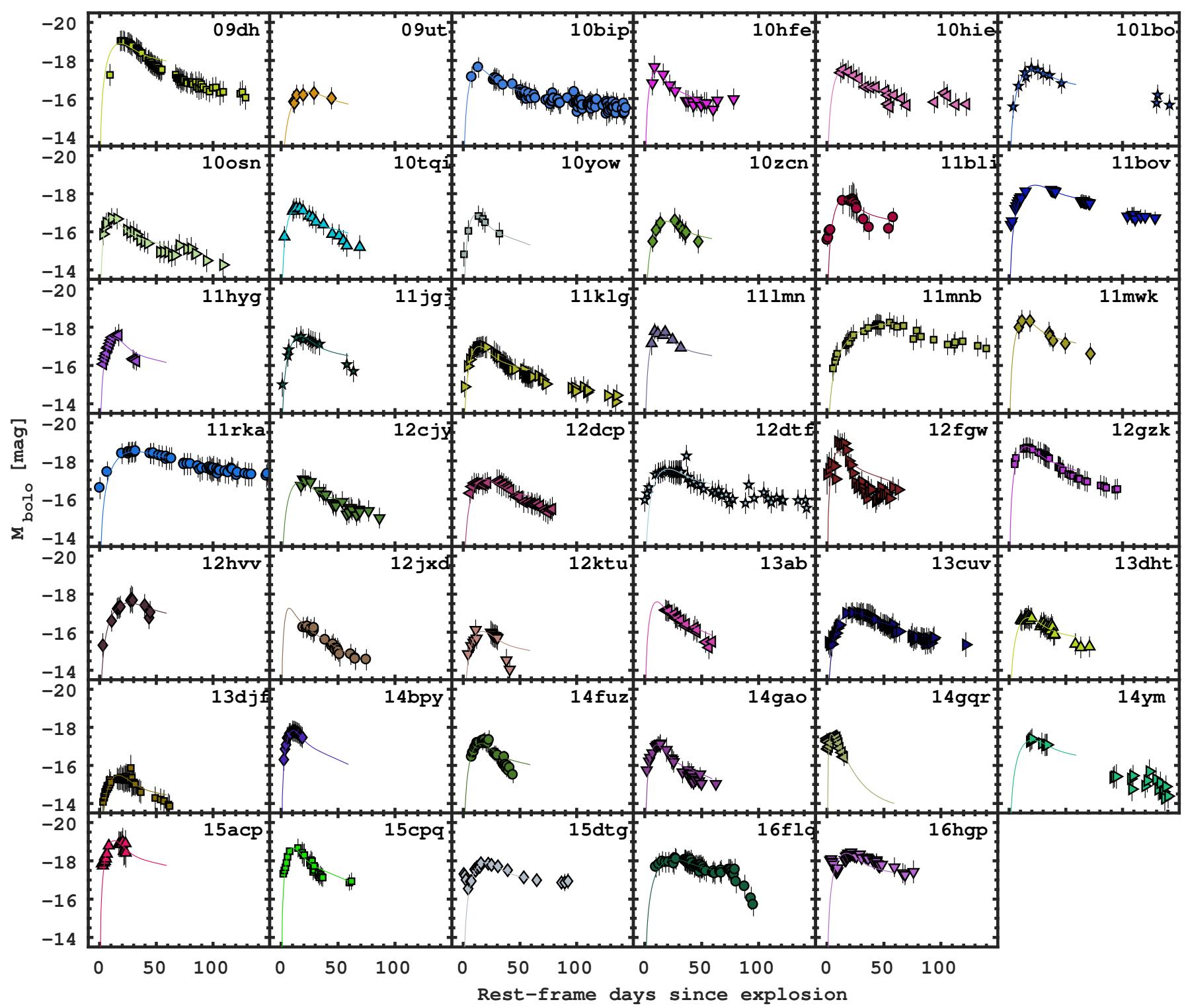

Fig. 18. Bolometric light curve computed and fitted with Arnett model for the $41 \mathrm{SNe}$ of the sample.

was most likely due to an extended envelope around the progenitor star, which they claim was a massive $\left(>35 M_{\odot}\right)$ Wolf-Rayet star. The overall explosion parameters we estimated using the Arnett model are somewhat consistent with their lower values. In the subsequent paper, they accounted for additional peculiar behavior of the SN at late times, which was explained by a combination of radioactive and magnetar powering which leads to a lower estimate of $M_{\mathrm{ej}}$ when compared with our estimate.

iPTF14gqr was presented in De et al. (2018) where they concluded that the best interpretation for this fast event is an ultrastripped SN. We also obtained low values for the ejecta mass and kinetic energy, in agreement with the scenario presented in De et al. (2018). iPTF11mnb was presented in a separate paper as a SN Ic from a massive progenitor $\left(85 M_{\odot}\right.$; Taddia et al. 2018a). Our estimates also show high values for the explosion parameters pointing towards a massive progenitor star. iPTF12gty was classified as a SLSN by Quimby et al. (2018) and further investigated by De Cia et al. (2018). Our spectral classification was pointing towards a SN Ic classification but this SN is a clear outlier in the sample in many ways. In particular, when applying the Arnett fit to estimate the explosion parameters we get a very high value of the ${ }^{56} \mathrm{Ni}$ mass. We therefore conclude that iPTF12gty is most likely a super-luminous SN.

Our $r$-band absolute magnitudes span the interval -15.54 to $-19.81 \mathrm{mag}$, with an average of $\left\langle M_{r}\right\rangle=-17.71 \pm 0.85 \mathrm{mag}$. The ranges available in the literature are $M_{r}^{\text {peak }}=-18.26 \pm 0.21 \mathrm{mag}$ (Taddia et al. 2015); $M_{r}^{\text {peak }}=-17.64 \pm 0.26$ mag (Taddia et al. $2018 \mathrm{~b}$ ) and $M_{r}^{\text {peak }}=-18.3 \pm 0.6 \mathrm{mag}$ (Drout et al. 2011). The average peak magnitude in the $r$ band estimated for our sample is in agreement with the ones from literature. We compared our values also with the (i)PTF sample of SNe Ic-BL (Taddia et al. 2019 b) where the peak magnitudes show a brighter average of $-18.7 \pm 0.7 \mathrm{mag}$.

We analyzed the shapes of the $r$-band light curves and of the bolometric light curves, searching for correlations among the different parameters. We identified a correlation in the $r$ band between $\Delta m_{15}$ and $\Delta m_{-10}$ which is in agreement with the results from Taddia et al. (2019b) and Drout et al. (2011). We note that the fact that the fast risers are also the fast decliners is not trivially true. There could well be different physical circumstances determining the rise and the decline from peak, for example the 
Table 5. Estimated Fe II $\lambda 5169$ velocities at peak for $44 \mathrm{SNe}$ of the sample.

\begin{tabular}{|c|c|}
\hline $\mathrm{SN}$ & $\begin{array}{c}v_{\max } \\
\left(\mathrm{km} \mathrm{s}^{-1}\right)\end{array}$ \\
\hline 09dh & 8125.6 \\
\hline 09ut & 8125.6 \\
\hline 10bip & 13706.70 \\
\hline $10 \mathrm{hfe}$ & 9497.55 \\
\hline 10hie & 10108.39 \\
\hline 10lbo & 9140.21 \\
\hline 10osn & 4589.67 \\
\hline 10tqi & 9315.55 \\
\hline 10yow & 9464.53 \\
\hline $10 \mathrm{zcn}$ & 8819.06 \\
\hline $11 \mathrm{bli}$ & 4847.10 \\
\hline $11 \mathrm{bov}$ & 5815.18 \\
\hline 11 hyg & 7832.18 \\
\hline $11 \mathrm{klg}$ & 8172.07 \\
\hline $11 \mathrm{jgj}$ & 8125.6 \\
\hline $11 \mathrm{lmn}$ & 2437.49 \\
\hline $11 \mathrm{mnb}$ & 5005.96 \\
\hline $11 \mathrm{mwk}$ & 8573.56 \\
\hline $11 \mathrm{rka}$ & 5739.87 \\
\hline $12 \mathrm{cjy}$ & 8125.6 \\
\hline $12 \mathrm{dcp}$ & 6493.21 \\
\hline $12 \mathrm{dtf}$ & 8894.58 \\
\hline $12 \mathrm{fgw}$ & 8125.6 \\
\hline 12 gty & 3492.84 \\
\hline $12 \mathrm{gzk}$ & 16984.08 \\
\hline $12 \mathrm{hvV}$ & 8076.82 \\
\hline $12 \mathrm{jxd}$ & 7831.30 \\
\hline $12 \mathrm{ktu}$ & 8319.47 \\
\hline $13 a b$ & 11149.58 \\
\hline $13 \mathrm{aot}$ & 7728.21 \\
\hline $13 \mathrm{cuv}$ & 5389.32 \\
\hline $13 \mathrm{dht}$ & 7065.56 \\
\hline $14 \mathrm{djf}$ & 8125.6 \\
\hline $14 \mathrm{bpy}$ & 11148.79 \\
\hline $14 \mathrm{fuz}$ & 9754.77 \\
\hline $14 \mathrm{gao}$ & 10171.24 \\
\hline $14 \mathrm{gqr}$ & 10472.35 \\
\hline $14 \mathrm{jhf}$ & 7989.49 \\
\hline $14 y m$ & 7436.66 \\
\hline $15 \mathrm{acp}$ & 6570.15 \\
\hline $15 \mathrm{cpq}$ & 9960.42 \\
\hline $15 \mathrm{dtg}$ & 8261.14 \\
\hline $16 f l q$ & 6281.47 \\
\hline 16hgp & 6235.76 \\
\hline
\end{tabular}

mixing out of radioactive nickel will affect the steepness of the rising light curve whereas the time scale for the decline may be more determined by the ejecta mass and composition. We did not find any Phillips-like relation and this is in agreement with previous works (Taddia et al. 2019b; Lyman et al. 2016; Drout et al. 2011).

We compared the estimated average values for the explosion parameters of the 41 (i)PTF SNe Ic with the ones available in the literature. Drout et al. (2011) presented $M_{56} \mathrm{Ni}$ values for 9 SNe Ic. In Cano (2013) the explosion parameters for $13 \mathrm{SNe}$ Ic are presented. Taddia et al. (2015) analyzed three events, while the Lyman et al. (2016) sample contains 8 SNe Ic. A total num-
Table 6. Estimated explosion parameters for the 42 SNe. PTF12gty estimated explosion parameters are presented here in the table for completeness but this $\mathrm{SN}$ is excluded in the analysis.

\begin{tabular}{|c|c|c|c|}
\hline $\mathrm{SN}$ & $\begin{array}{c}M_{\mathrm{ej}} \\
\left(M_{\odot}\right)\end{array}$ & $\begin{array}{c}E_{\mathrm{K}} \\
\left(10^{51} \mathrm{erg}\right)\end{array}$ & $\begin{array}{l}M_{56} \mathrm{Ni} \\
\left(M_{\odot}\right) \\
\end{array}$ \\
\hline 09dh & $4.83(1.92)$ & $1.90(0.38)$ & $0.56(0.06)$ \\
\hline 09ut & $8.65(3.44)$ & $3.41(0.68)$ & $0.07(0.01)$ \\
\hline 10bip & $3.99(1.59)$ & $4.47(0.89)$ & $0.13(0.01)$ \\
\hline $10 \mathrm{hfe}$ & $1.23(0.49)$ & $0.66(0.13)$ & $0.09(0.01)$ \\
\hline 10hie & $3.19(1.27)$ & $1.95(0.39)$ & $0.12(0.01)$ \\
\hline $10 \mathrm{lbo}$ & $7.59(3.02)$ & $3.78(0.76)$ & $0.18(0.02)$ \\
\hline 10 osn & $0.58(0.23)$ & $0.07(0.01)$ & $0.05(0.01)$ \\
\hline 10tqi & $2.49(0.99)$ & $1.29(0.26)$ & $0.09(0.01)$ \\
\hline 10yow & $1.61(0.64)$ & $0.86(0.17)$ & $0.06(0.01)$ \\
\hline $10 \mathrm{zcn}$ & $5.31(2.11)$ & $2.47(0.49)$ & $0.07(0.01)$ \\
\hline $11 \mathrm{bli}$ & $1.82(0.72)$ & $0.26(0.05)$ & $0.16(0.02)$ \\
\hline $11 \mathrm{bov}$ & $5.78(2.30)$ & $1.17(0.23)$ & $0.46(0.05)$ \\
\hline 11hyg & $2.10(0.83)$ & $0.77(0.15)$ & $0.11(0.01)$ \\
\hline $11 \mathrm{jgj}$ & $3.97(1.58)$ & $1.56(0.31)$ & $0.15(0.01)$ \\
\hline $11 \mathrm{klg}$ & $2.54(1.01)$ & $1.01(0.20)$ & $0.08(0.01)$ \\
\hline $111 \mathrm{mn}$ & $0.56(0.22)$ & $0.02(0.01)$ & $0.15(0.01)$ \\
\hline $11 \mathrm{mnb}$ & $31.29(12.45)$ & $4.68(0.94)$ & $0.74(0.08)$ \\
\hline $11 \mathrm{mwk}$ & $2.77(1.10)$ & $1.22(0.24)$ & $0.28(0.03)$ \\
\hline 11rka & $11.05(4.40)$ & $2.17(0.43)$ & $0.63(0.06)$ \\
\hline $12 \mathrm{cjy}$ & $3.77(1.50)$ & $1.49(0.30)$ & $0.08(0.01)$ \\
\hline $12 \mathrm{dcp}$ & $4.15(1.65)$ & $1.05(0.21)$ & $0.10(0.01)$ \\
\hline $12 \mathrm{dtf}$ & $7.04(2.80)$ & $3.32(0.66)$ & $0.19(0.02)$ \\
\hline $12 \mathrm{fgw}$ & $1.18(0.47)$ & $0.46(0.09)$ & $0.24(0.02)$ \\
\hline $12 \mathrm{gzk}$ & $6.31(2.51)$ & $10.86(2.17)$ & $0.41(0.04)$ \\
\hline 12 gty $^{(a)}$ & $21.42(8.52)$ & $1.56(0.31)$ & $2.98(0.30)$ \\
\hline $12 \mathrm{hvv}$ & $10.30(4.10)$ & $4.01(0.80)$ & $0.22(0.02)$ \\
\hline $12 \mathrm{jxd}$ & $0.42(0.17)$ & $0.15(0.03)$ & $0.06(0.01)$ \\
\hline $12 \mathrm{ktu}$ & $4.02(1.60)$ & $1.66(0.33)$ & $0.04(0.02)$ \\
\hline $13 a b$ & $1.68(0.67)$ & $1.25(0.25)$ & $0.11(0.01)$ \\
\hline $13 \mathrm{cuv}$ & $5.19(2.06)$ & $0.90(0.18)$ & $0.12(0.01)$ \\
\hline $13 \mathrm{dht}$ & $3.43(1.36)$ & $1.02(0.20)$ & $0.07(0.01)$ \\
\hline $13 \mathrm{djf}$ & $3.78(1.50)$ & $1.49(0.30)$ & $0.02(0.01)$ \\
\hline 14bpy & $1.63(0.65)$ & $1.21(0.24)$ & $0.13(0.01)$ \\
\hline $14 \mathrm{fuz}$ & $3.76(1.49)$ & $2.13(0.43)$ & $0.10(0.01)$ \\
\hline $14 \mathrm{gao}$ & $1.42(0.56)$ & $0.87(0.17)$ & $0.06(0.01)$ \\
\hline $14 \mathrm{gqr}$ & $0.12(0.05)$ & $0.08(0.02)$ & $0.06(0.01)$ \\
\hline $14 y m$ & $5.79(2.30)$ & $1.91(0.38)$ & $0.15(0.02)$ \\
\hline $15 \mathrm{acp}$ & $1.62(0.64)$ & $0.42(0.08)$ & $0.47(0.05)$ \\
\hline $15 \mathrm{cpq}$ & $1.43(0.57)$ & $0.85(0.17)$ & $0.28(0.03)$ \\
\hline $15 \mathrm{dtg}$ & $5.69(2.26)$ & $2.32(0.46)$ & $0.24(0.02)$ \\
\hline $16 f l q$ & 7.64(3.04) & $1.80(0.36)$ & $0.36(0.04)$ \\
\hline $16 \mathrm{hgp}$ & $3.01(1.20)$ & $0.70(0.14)$ & $0.33(0.03)$ \\
\hline
\end{tabular}

Notes. ${ }^{(a)}$ PTF12gty estimated explosion parameters are presented here in the table for completeness but this SN is excluded in the analysis.

ber of 13 SNe Ic was presented in Prentice et al. (2016). Taddia et al. (2018b) presented 11 SNe Ic and in Prentice et al. (2019) three SNe Ic are included. Our (i)PTF sample with 41 SNe Ic therefore by far represents the largest sample of $\mathrm{SNe}$ Ic available where the explosion parameters have been estimated. We estimated the cumulative distribution functions (CDF) of the explosions parameters, and compared it to the available studies in the literature. The results of these comparisons are shown in Fig. 21.

We also report the average values and their standard deviations for the estimated explosion parameters from the different samples in Table 7. We also compared the $M_{56 \mathrm{Ni}}$ estimated by 

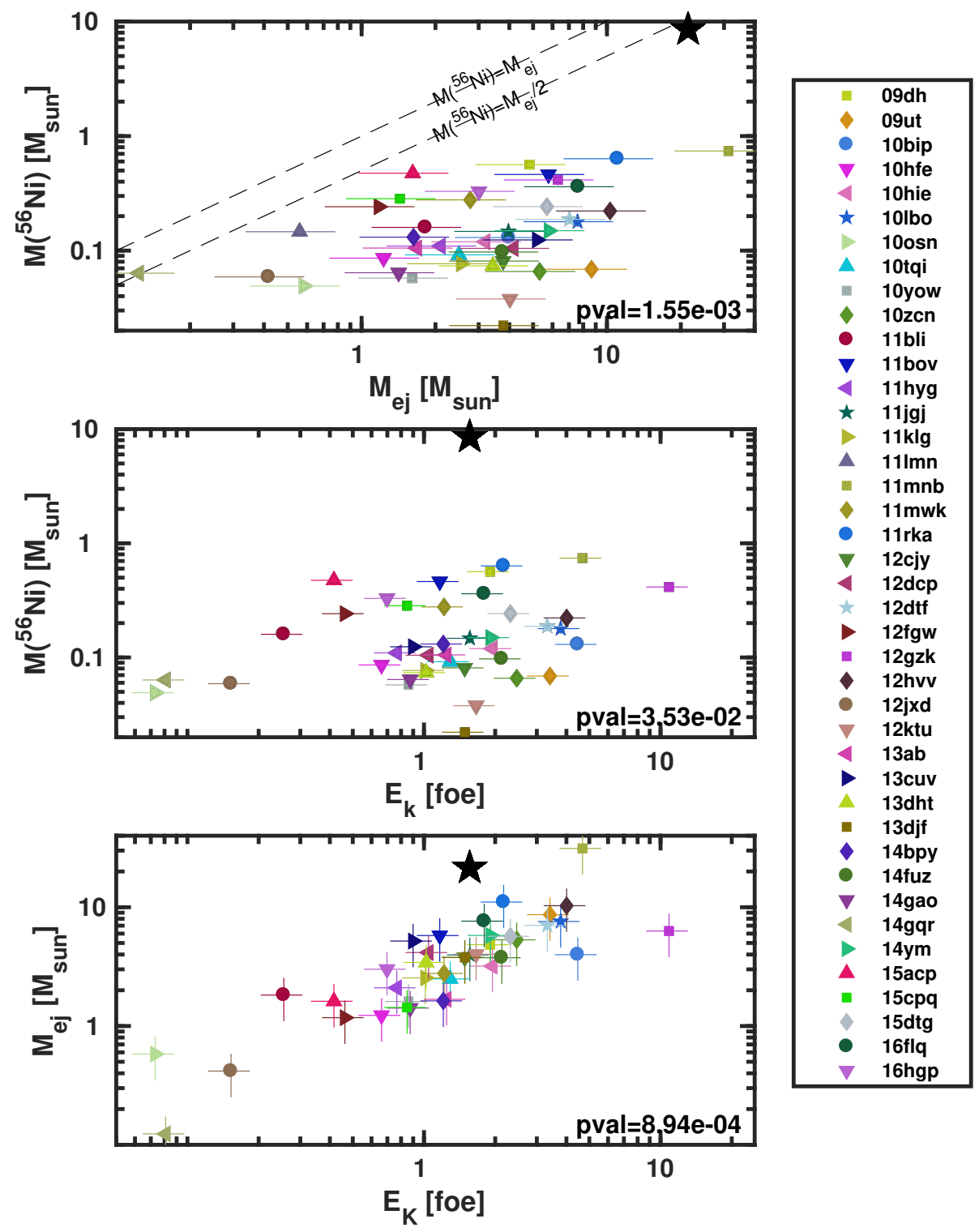

Fig. 19. Explosions parameters for $41 \mathrm{SNe}$ Ic plotted against each other. We see clear correlations between the parameters, as quantified by the $p$-values in the panels. SN PTF12gty has also been represented for completeness with a black star.
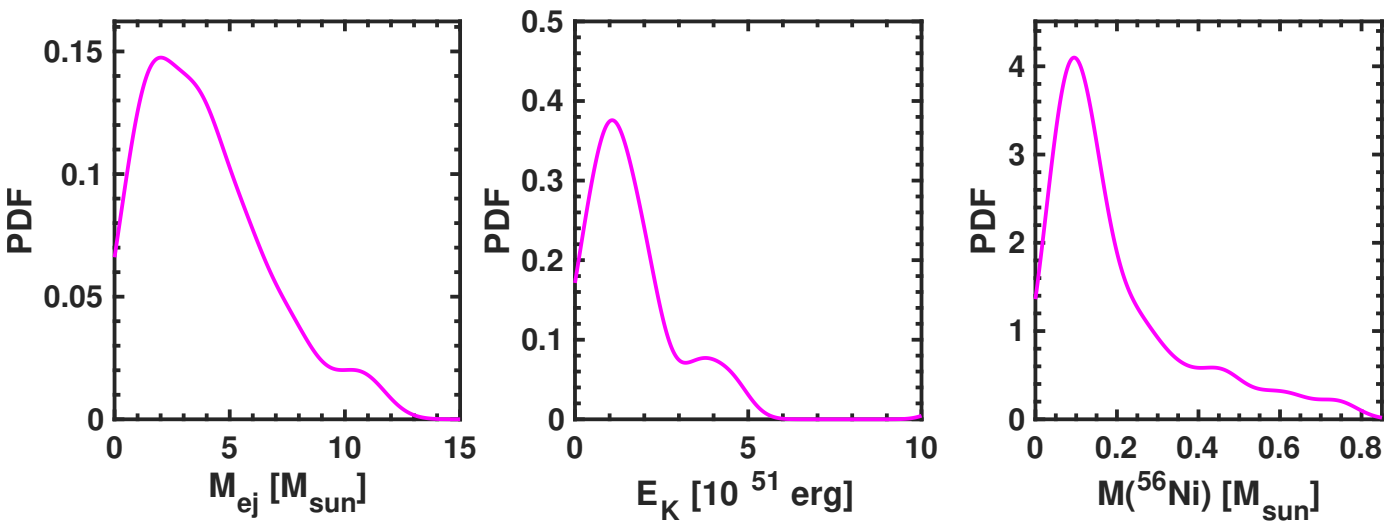

Fig. 20. Probability density functions for explosion parameters for our sample of SNe Ic.

Meza \& Anderson (2020) for 6 SNe Ic using Arnett model, and they get an average value lower than the one found in this work.

We searched for correlations among the explosions parameters (see Fig. 19), and identify a correlation between $M_{\mathrm{ej}}$ and $E_{\mathrm{K}}$. We also notice correlations between $M_{\mathrm{ej}}$ and $M_{56 \mathrm{Ni}}$, and between
$M_{56 \mathrm{Ni}}$ and $E_{\mathrm{K}}$. These correlations were also observed in other SE SN studies (Taddia et al. 2019a, 2018a; Lyman et al. 2016). The strong correlation between ejecta mass and kinetic energy is, just as noted by Lyman et al. (2016), in fact mainly driven by the ejecta mass. The range in ejecta mass is much larger than the 


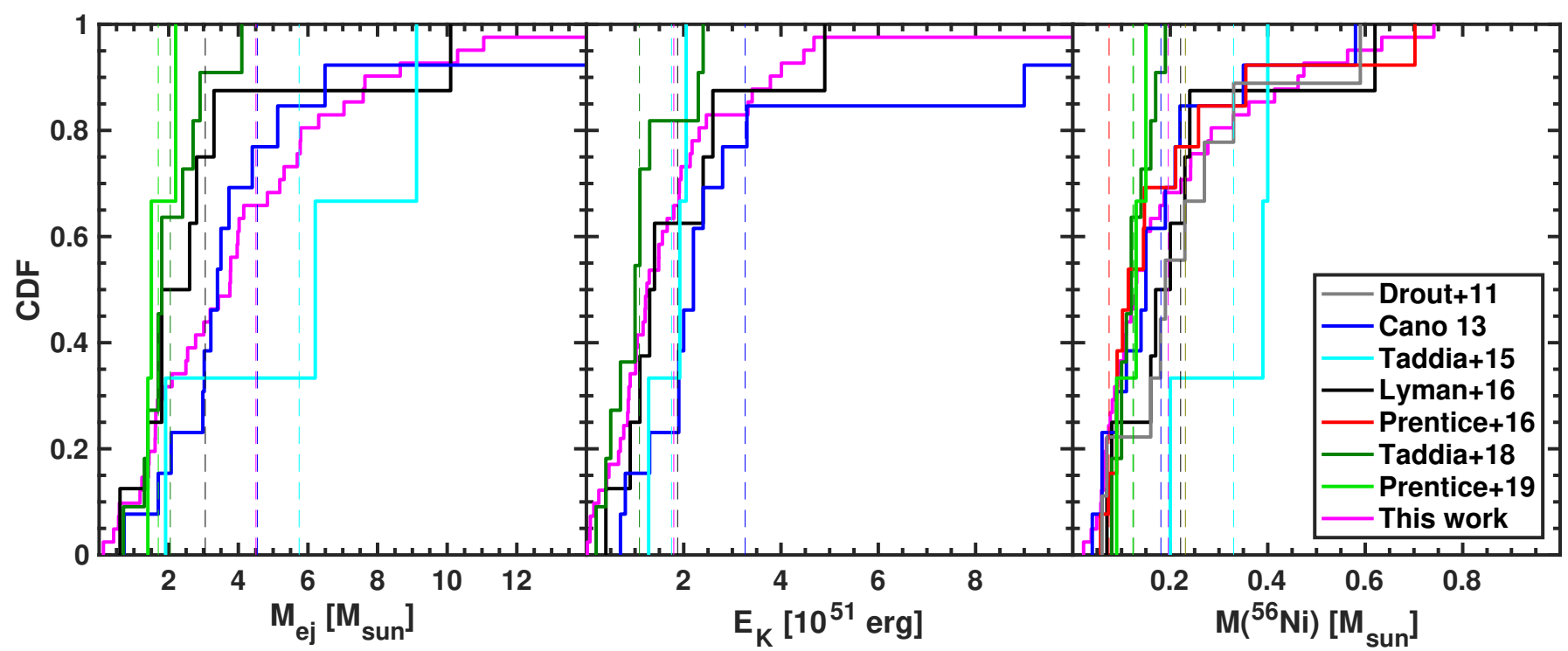

Fig. 21. Cumulative distribution functions for the explosion parameters compared to those of other samples in the literature. Dashed lines represent the average values for each sample from literature.

Table 7. Comparison of the average estimates of the explosions parameters with estimates from the literature.

\begin{tabular}{lccc}
\hline \hline & $\begin{array}{c}M_{\mathrm{ej}} \\
\left(M_{\odot}\right)\end{array}$ & $\begin{array}{c}E_{\mathrm{K}} \\
\left(10^{51} \mathrm{erg}\right)\end{array}$ & $\begin{array}{c}M_{56 \mathrm{Ni}} \\
\left(M_{\odot}\right)\end{array}$ \\
\hline Drout+11 & $1.7_{-0.9}^{+1.4}$ & $1.0_{-0.5}^{+0.9}$ & $0.24(0.15)$ \\
Taddia+15 & $5.7(3.6)$ & $1.7(0.4)$ & $0.33(0.11)$ \\
Lyman+16 & $3.0(2.8)$ & $1.9(1.3)$ & $0.22(0.16)$ \\
Prentice+16 & $\ldots$ & $\ldots$ & $0.16_{-0.10}^{+0.03}$ \\
Taddia+18 & $2.1(1.0)$ & $1.2(0.7)$ & $0.13(0.04)$ \\
Prentice+19 & $3.0(0.7)$ & $\ldots$ & $0.11(0.09)$ \\
This work $^{(a)}$ & $4.50(0.79)$ & $1.79(0.29)$ & $0.19(0.03)$ \\
This work $^{(b)}$ & $3.57(0.40)$ & $1.74(0.33)$ & $0.16(0.02)$ \\
This work $^{(c)}$ & $3.67(0.39)$ & $1.78(0.32)$ & $0.16(0.02)$ \\
This work $^{(d)}$ & $4.65(0.92)$ & $1.88(0.34)$ & $0.19(0.03)$ \\
\hline
\end{tabular}

Notes. ${ }^{(a)}$ Average values for the overall sample of 41 SNe. ${ }^{(b)}$ Average values when the $5 \mathrm{SNe}$ with broad light curve are excluded. ${ }^{(c)}$ Average values when the $5 \mathrm{SNe}$ with broad light curve and the 1 fast $\mathrm{SN}$ are excluded. ${ }^{(d)}$ Average values when the $7 \mathrm{SNe}$ with Ibc classification are excluded.

variation in velocity, and this is driving the relation. There is no correlation between ejecta mass and photosperic velocity.

\subsection{Implications for progenitors}

The PDF of the different explosion parameters are shown in Fig. 20. The $E_{\mathrm{K}}$ shows a first strong peak for energies lower than 3 foe and the $M_{56 \mathrm{Ni}}$ distribution shows a clear peak at values lower than $0.3 M_{\odot}$. The PDF of the $M_{\text {ej }}$ shows a first peak for values lower than $5 M_{\odot}$ and shows indication for additional peak(s) towards higher mass. A similar analysis for $M_{\mathrm{ej}}$ was presented in Lyman et al. (2016) and from a comparison with expectations from models they concluded that since the peak of ejecta masses is rather low, this indicates that the majority of SE SNe originate from not too massive stars, assuming the remnant is a neutron star. Moreover, since such stars are unable to get rid of all of their outer hydrogen and helium layers solely from massloss from winds, they must likely have been born and stripped in a binary system. The trend we see here for our larger sample would lead to a similar conclusion. We notice that the PDF presented in Lyman et al. (2016) did not show a very pronounced secondary peak. This is due to the presence of SNe with broad light curves in our sample, which could arise from more massive star progenitors. We note that the apparent secondary peak in the PDF at $\sim 10 M_{\odot}$ of ejected mass seems to be compatible with single, massive WR progenitors. These $\mathrm{SNe}$ will be discussed into more detail by Karamemehtoglu et al. (in prep.).

We have presented the sample of Type Ic supernovae collected by (i)PTF over a period of $\sim 7$ years. The final sample of $\mathrm{SNe}$ that also have pre-peak photometry is made up of 44 objects, which we analyze in terms of light-curve and explosion parameters. This is the largest such sample to date. Our main results confirm trends seen in previous articles based on smaller and less homogeneous samples. Although our data are not always fantastic for individual SNe, the bulk sample provides a good picture of the overall properties of this class of extremely stripped supernovae.

The moderate ejecta masses remain a challenge for scenarios involving single very massive stars, as already proposed by Lyman et al. (2016), and corroborate discussions on the need for binary star evolution to produce most of the Type Ic SNe. Indications for a population of more massive progenitors are also seen. The ejected masses of radioactive nickel are $\sim 0.2 M_{\odot}$, which is more than current neutrino driven explosion models (Ertl et al. 2020) can easily accomplish. As mentioned, the correlation between ejecta mass and energy is largely spurious but the correlation between ejecta mass and mass of radioactive nickel appears to be robust. It is statistically significant even if we exclude the most massive object that drives the correlation, and is something that a generic explosion model would have to explain.

There is hope for better understanding of these explosions from the observational perspective. The Zwicky Transient Facility (Bellm et al. 2019) that has taken over on the P48 telescope after (i)PTF enable superior light curves also of Type Ic SNe. Over the first years, this survey has already observed almost 100 Type Ic SNe, and a fair fraction of these have better 
sampled LC:s than the sample we have presented here. We look forward to analyzing these new data.

Acknowledgements. The Oskar Klein Centre was funded by the Swedish Research Council. C.B. gratefully acknowledges support from the Knut and Alice Wallenberg Foundation, and from the Wennergren Foundation (PI JS). The intermediate Palomar Transient Factory project is a scientific collaboration among the California Institute of Technology, Los Alamos National Laboratory, the University of Wisconsin, Milwaukee, the Oskar Klein Center, the Weizmann Institute of Science, the TANGO Program of the University System of Taiwan, and the Kavli Institute for the Physics and Mathematics of the Universe. LANL participation in iPTF is supported by the US Department of Energy as a part of the Laboratory Directed Research and Development program. The data presented herein were obtained in part with ALFOSC, which is provided by the Instituto de Astrofisica de Andalucia (IAA) under a joint agreement with the University of Copenhagen and NOTSA. IA is a CIFAR Azrieli Global Scholar in the Gravity and the Extreme Universe Program and acknowledges support from that program, from the Israel Science Foundation (grant numbers 2108/18 and 2752/19), from the United States - Israel Binational Science Foundation (BSF), and from the Israeli Council for Higher Education Alon Fellowship. AGY's research is supported by the EU via ERC grant No. 725161, the ISF GW excellence center, an IMOS space infrastructure grant and BSF/Transformative and GIF grants, as well as The Benoziyo Endowment Fund for the Advancement of Science, the Deloro Institute for Advanced Research in Space and Optics, The Veronika A. Rabl Physics Discretionary Fund, Paul and Tina Gardner, Yeda-Sela and the WIS-CIT joint research grant; AGY is the recipient of the Helen and Martin Kimmel Award for Innovative Investigation.

\section{References}

Ahn, C. P., Alexandroff, R., Allende Prieto, C., et al. 2014, ApJS, 211, 17 Arnett, W. D. 1982, ApJ, 253, 785

Barbarino, C., Botticella, M. T., Dall'Ora, M., et al. 2017, MNRAS, 471, 2463

Begelman, M. C., \& Sarazin, C. L. 1986, ApJ, 302, L59

Bellm, E. C., Kulkarni, S. R., Graham, M. J., et al. 2019, PASP, 131, 018002

Ben-Ami, S., Gal-Yam, A., Filippenko, A. V., et al. 2012, ApJ, 760, L33

Bersten, M. C., Benvenuto, O. G., Folatelli, G., et al. 2014, AJ, 148, 68

Blondin, S., \& Tonry, J. L. 2007, ApJ, 666, 1024

Cano, Z. 2013, MNRAS, 434, 1098

Cao, Y., Kasliwal, M. M., Iair, A., et al. 2013, ApJ, 775, L7

Cenko, S. B., Fox, D. B., Moon, D.-S., et al. 2006, PASP, 118, 1396

Contardo, G., Leibundgut, B., \& Vacca, W. D. 2000, A\&A, 359, 876

Corsi, A., Ofek, E. O., Gal-Yam, A., et al. 2012, ApJ, 747, L5

De, K., Kasliwal, M. M., Ofek, E. O., et al. 2018, Science, 362, 201

De Cia, A., Gal-Yam, A., Rubin, A., et al. 2018, ApJ, 860, 100

Drout, M. R., Soderberg, A. M., Gal-Yam, A., et al. 2011, ApJ, 741, 97

Eldridge, J. J., Fraser, M., Smartt, S. J., Maund, J. R., \& Crockett, R. M. 2013,

MNRAS, 436, 774
Eldridge, J. J., Fraser, M., Maund, J. R., \& Smartt, S. J. 2015, MNRAS, 446, 2689

Ertl, T., Woosley, S. E., Sukhbold, T., \& Janka, H.-T. 2020, ApJ, 890, 51 Filippenko, A. V. 1997, ARA\&A, 35, 309

Fitzpatrick, E. L. 1999, PASP, 111, 63

Fremling, C., Sollerman, J., Taddia, F., et al. 2014, A\&A, 565, A114

Fremling, C., Sollerman, J., Taddia, F., et al. 2016, A\&A, 593, A68

Fremling, C., Sollerman, J., Kasliwal, M. M., et al. 2018, A\&A, 618, A37

Gal-Yam, A. 2017, Handbook of Supernovae (New York: Springer International Publishing AG), 195

Horesh, A., Kulkarni, S. R., Corsi, A., et al. 2013, ApJ, 778, 63

Karamehmetoglu, E., Taddia, F., Sollerman, J., et al. 2017, A\&A, 602, A93

Komatsu, E., Dunkley, J., Nolta, M. R., et al. 2009, ApJS, 180, 330

Kulkarni, S. R. 2013, ATel, 4807, 1

Law, N. M., Kulkarni, S. R., Dekany, R. G., et al. 2009, PASP, 121, 1395

Lyman, J. D., Bersier, D., \& James, P. A. 2014, MNRAS, 437, 3848

Lyman, J. D., Bersier, D., James, P. A., et al. 2016, MNRAS, 457, 328

Masci, F. J., Laher, R. R., Rebbapragada, U. D., et al. 2017, PASP, 129

Meza, N., \& Anderson, J. P. 2020, A\&A, 641, A177

Modjaz, M., Blondin, S., Kirshner, R. P., et al. 2014, AJ, 147, 99

Modjaz, M., Liu, Y. Q., Bianco, F. B., \& Graur, O. 2016, ApJ, 832, 108

Modjaz, M., Bianco, F. B., Siwek, M., et al. 2020, ApJ, 892, 153

Mould, J. R., Huchra, J. P., Freedman, W. L., et al. 2000a, ApJ, 529, 786

Mould, J. R., Huchra, J. P., Freedman, W. L., et al. 2000b, ApJ, 545, 547

Nyholm, A., Sollerman, J., Tartaglia, L., et al. 2020, A\&A, 637, A73

Ofek, E. O., Laher, R., Law, N., et al. 2012, PASP, 124, 62

Phillips, M. M. 1993, ApJ, 413, L105

Pastorello, A., Smartt, S. J., Botticella, M. T., et al. 2010, ApJ, 724, L16

Podsiadlowski, P., Joss, P. C., \& Hsu, J. J. L. 1992, ApJ, 391, 246

Poznanski, D., Prochaska, J. X., \& Bloom, J. S. 2012, MNRAS, 426, 1465

Prentice, S. J., Mazzali, P. A., Pian, E., et al. 2016, MNRAS, 458, 2973

Prentice, S. J., Ashall, C., James, P. A., et al. 2019, MNRAS, 485, 1559

Quimby, R. M., De Cia, A., Gal-Yam, A., et al. 2018, ApJ, 855, 2

Rahmer, G., Smith, R., Velur, V., et al. 2008, Ground-based and Airborne Instrumentation for Astronomy II, Proc. SPIE, 7014

Rau, A., Kulkarni, S. R., Law, N. M., et al. 2009, PASP, 121, 1334

Schlafly, E. F., \& Finkbeiner, D. P. 2011, ApJ, 737, 103

Smartt, S. J. 2009, ARA\&A, 47, 63

Stritzinger, M. D., Taddia, F., Burns, C. R., et al. 2018, A\&A, 609, A135

Taddia, F., Sollerman, J., Leloudas, G., et al. 2015, A\&A, 574, A60

Taddia, F., Fremling, C., Sollerman, J., et al. 2016, A\&A, 592, A89

Taddia, F., Sollerman, J., Fremling, C., et al. 2018a, A\&A, 609, A106

Taddia, F., Stritzinger, M. D., Bersten, M., et al. 2018b, A\&A, 609, A136

Taddia, F., Sollerman, J., Fremling, C., et al. 2019a, A\&A, 621, A64

Taddia, F., Sollerman, J., Fremling, C., et al. 2019b, A\&A, 621, A71

Taubenberger, S., Pastorello, A., Mazzali, P. A., et al. 2006, MNRAS, 371, 1459

Valenti, S., Benetti, S., Cappellaro, E., et al. 2008, MNRAS, 383, 1485

Valenti, S., Taubenberger, S., Pastorello, A., et al. 2012, ApJ, 749, L28

Wheeler, J. C., \& Levreault, R. 1985, ApJ, 294, L17

Woosley, S. E., Langer, N., \& Weaver, T. A. 1995, ApJ, 448, 315

Yaron, O., \& Gal-Yam, A. 2012, PASP, 124, 668

Yoon, S.-C., Woosley, S. E., \& Langer, N. 2010, ApJ, 725, 940 